\title{
Optimisation of the HS-SPME/GC-MS Approach by Design of Experiments Combined with Chemometrics for the Classification of Cretan Virgin Olive Oils
}

\author{
Artemis Lioupi ${ }^{1,2,3}$, Ioannis Sampsonidis ${ }^{2,3,4}{ }^{-0}$, Christina Virgiliou ${ }^{1,2,3}$ (), Vassiliki T. Papoti ${ }^{5}$, \\ Kyriaki G. Zinoviadou ${ }^{5}$, Apostolos Spyros ${ }^{6}(\mathbb{D}$ and Georgios Theodoridis $1,2,3, * \mathbb{C}$
}

Citation: Lioupi, A.; Sampsonidis, I.; Virgiliou, C.; Papoti, V.T.; Zinoviadou, K.G.; Spyros, A.; Theodoridis, G. Optimisation of the HS-SPME/GCMS Approach by Design of Experiments Combined with Chemometrics for the Classification of Cretan Virgin Olive Oils.

Metabolites 2022, 12, 114. https:// doi.org/10.3390/metabo12020114

Academic Editor: Federico Tesio Torta

Received: 5 January 2022

Accepted: 20 January 2022

Published: 25 January 2022

Publisher's Note: MDPI stays neutral with regard to jurisdictional claims in published maps and institutional affiliations.

Copyright: (C) 2022 by the authors. Licensee MDPI, Basel, Switzerland. This article is an open access article distributed under the terms and conditions of the Creative Commons Attribution (CC BY) license (https:// creativecommons.org/licenses/by/ $4.0 /)$.
1 Laboratory of Analytical Chemistry, School of Chemistry, Aristotle University of Thessaloniki, GR-54124 Thessaloniki, Greece; liouarte@chem.auth.gr (A.L.); cr_virgi@hotmail.com (C.V.)

2 Biomic AUTh, Center for Interdisciplinary Research and Innovation (CIRI-AUTH), Balkan Center B1.4, 10th km Thessaloniki-Thermi Rd, P.O. Box 8318, GR-57001 Thessaloniki, Greece; isampsonides@yahoo.gr

3 FoodOmicsGR Research Infrastructure, AUTh Node, Center for Interdisciplinary Research and Innovation (CIRI-AUTH), Balkan Center B1.4, 10th km Thessaloniki-Thermi Rd, P.O. Box 8318, GR-57001 Thessaloniki, Greece

4 Department of Nutritional Sciences and Dietetics, International Hellenic University, GR-57400 Thessaloniki, Greece

5 Department of Food Science and Technology, Perrotis College, American Farm School, GR-55102 Thessaloniki, Greece; vpapot@afs.edu.gr (V.T.P.); kzinov@afs.edu.gr (K.G.Z.)

6 NMR Laboratory, Department of Chemistry, University of Crete, Voutes Campus, P.O. Box 2208, GR-71003 Heraklion, Crete, Greece; aspyros@chemistry.uoc.gr

* Correspondence: gtheodor@chem.auth.gr

\begin{abstract}
A headspace-solid phase microextraction/gas chromatography-mass spectrometry (HSSPME/GC-MS) method was developed herein for the analysis of virgin olive oil volatile metabolome. Optimisation of SPME conditions was performed by Design of Experiments (DoE) and Response Surface Methodology (RSM) approaches and factors, such as sample volume, sample stirring, extraction temperature and time, and desorption temperature and time, were examined to reach optimal microextraction conditions. The potential of the optimised method was then investigated for its use in the classification of Cretan virgin olive oil samples with the aid of multivariate statistical analysis. Certain markers were identified with significance in the geographical classification of Cretan extra-virgin olive oil (EVOO) samples. In total, 92 volatile organic compounds were tentatively identified and semi-quantified, and the data obtained confirm that the method is robust, reliable, and analytically powerful for olive oil classification.
\end{abstract}

Keywords: gas chromatography-mass spectrometry (GC-MS); headspace-solid phase microextraction (HS-SPME); olive oil; volatile organic compounds (VOCs); volatilomics; foodomics; metabolomics

\section{Introduction}

Extra virgin olive oil is one of the most significant ingredients of the Mediterranean diet, and its consumption has been significantly increased due to its high and special nutritional and sensory characteristics [1]. Virgin olive oil (VOO) is classified to quality categories as described by official standards (e.g., Codex Alimentarius, International Olive Council, and European Union) where sensory assessment is also included [2]. The sensory quality of $\mathrm{VOO}$ is determined by a panel test that evaluates positive and negative descriptors, according to European Union (EU) criteria (EU regulations) [3,4]. Olive oils possessing the fruity attribute and lacking any sensory defects can be categorized as "extra virgin", whilst the existence and severity of sensory defects are used to verify whether the oil could be categorized as "virgin" or lampante [5]. Volatile compounds in olive oil can be linked to both positive and negative sensory characteristics and have a major effect on the oil's 
quality and, as a result, on consumer preference. Hence, determining the VOO volatile fraction is important to ensure consumer protection, but also to avoid unfair competition, which can destabilize the market and disrupt regional/national economies.

In Greece, over 30 olive varieties are mainly produced [6]. Although Greece ranks third in the world in VOO production, the high-quality potential of Greek olive oils has been scarcely examined, and such information has not been communicated to the market. Crete is one of the main olive oil-producing areas in Greece and two oil cultivars, Koroneiki and Tsounati, mainly originate from there. Koroneiki, one of the most well-known and appraised olive cultivars in the world, is the predominant olive oil cultivar in Greece, accounting for about $60 \%$ of the country's olive oil production (approximately 250,000 $t$ ) [6]. The Tsounati variety (also referred to as "Athinolia" or "Mastoeidis") is less exploited and is cultivated in specific areas of Peloponnese, mainly in south Lakonia, Argolida, as well as in western Crete [7]. The quality potential of both examined cultivars can be partially revealed from the quality awards they gain from international competitions (e.g., Athena International Olive Oil Competition). It is necessary to verify the quality standards of Greek traditional high-added-value VOO to protect the final consumer from non-authentic food products and to help Greek food producers, suppliers, and service establishments compete in national and international markets [8-10].

Gas chromatography-mass spectrometry (GC-MS) has been commonly applied for the analysis of volatile organic compounds (VOCs) in EVOO, with an emphasis given in biomarker-detection, authenticity control, and issues associated with food quality and safety [11-13]. Headspace solid-phase microextraction (HS-SPME) has a number of advantages over other extraction methods, including automation and simplicity. Due to these advantages, it is usually the method of choice, with many applications in the field [14-16]. However, for this type of analysis, it is important to identify the role of all the possible factors that can influence the extraction of volatile compounds. In the present publication, sample volume, sample stirring, extraction temperature and time, and desorption time are the factors examined to reach optimal microextraction conditions. According to our knowledge, there are only two studies that focus on the optimisation of HS-SPME parameters by utilising advanced statistical techniques such as Design of Experiments (DOE) for VOO volatile compound analysis $[17,18]$. The presented approach is the first that optimises five HS-SPME factors simultaneously for EVOO analysis.

The overall goal was the development of an HS-SPME/GC-MS method for the analysis of a VOO volatile profile. For this purpose, an optimisation of SPME conditions was performed using DOE and Response Surface Methodology (RSM) approaches. The method was then applied for the analysis of VOOs originating from different regions of Crete, Southern Greece. This study was focused on the potential use of the optimised HS-SPME/GC-MS method combined with chemometrics for sample classification. The method proved to be robust, enabling the identification and semi-quantification of VOO VOCs.

\section{Results and Discussion}

\subsection{Optimisation of SPME Extraction Conditions}

SPME sorption is dependent among other parameters on the sample volume, and it has been observed that extraction efficiency is improved when the volume of HS in the vial is minimised [19]. However, the extraction efficiency of volatile compounds may show a non-linear relation toward the sample volume due to fiber overload. Hence, the sample mass of the sample was examined under four different quantities $(1.000,1.850,3.000$, and $4.000 \mathrm{~g}$ ). As illustrated in Figure 1a, sample amounts of 1.850 and $3.000 \mathrm{~g}$ improved extraction efficiency. However, a further increase from 3.000 to $4.000 \mathrm{~g}$ of sample had a negative effect on the extraction efficiency, yielding an optimal sample amount of $1.850 \mathrm{~g}$.

For the optimisation of stirring, four different stirring speeds $(100,150,200,250$, and $300 \mathrm{rpm}$ ) were evaluated. As shown in Figure 1b, after sample stirring at $250 \mathrm{rpm}$, no further improvement in extraction efficiency was observed. Hence, a stirring speed of $250 \mathrm{rpm}$ was used for further experiments. 


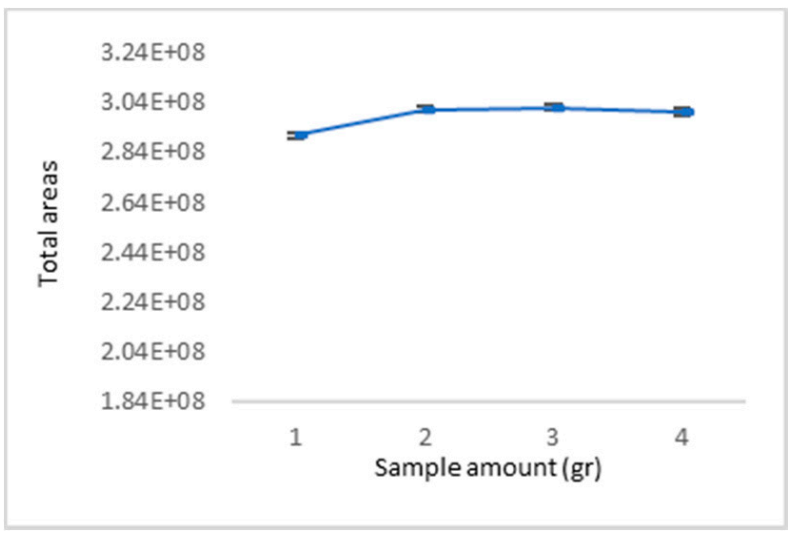

(a)

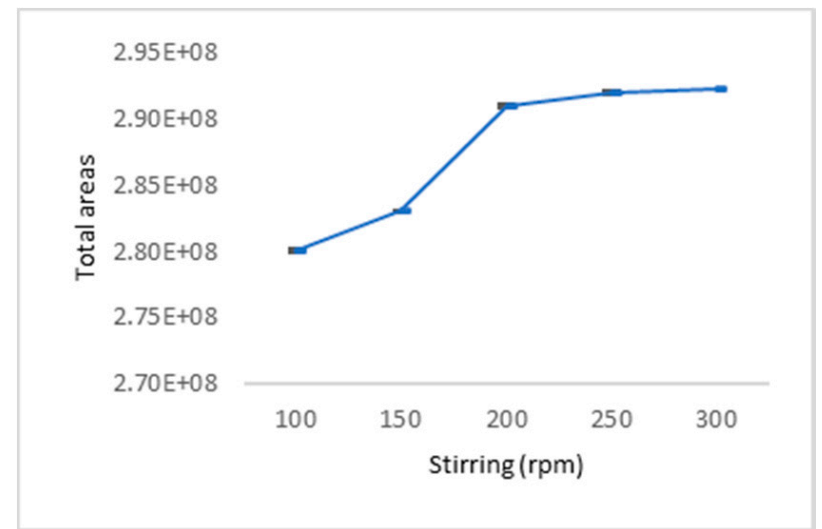

(b)

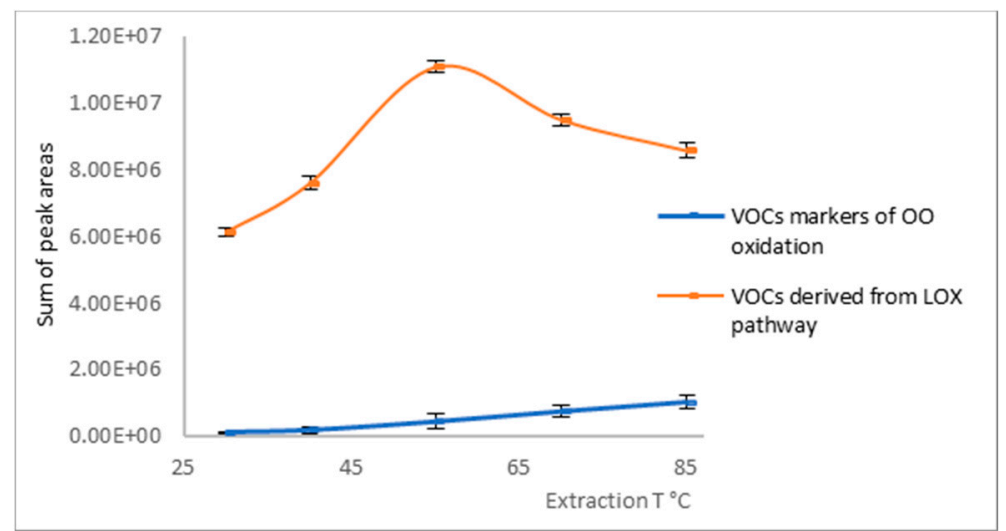

(c)

Figure 1. Influence of (a) sample amount, (b) stirring speed on total areas, and (c) extraction temperature on selected VOCs. $\left(\mathrm{E}+08=\times 10^{8}\right)$.

Although included in the DOE-RSM optimisation as a studied factor, a preliminary screening experiment was performed to determine the behaviour of the most critical HSSPME condition for EVOO analysis, which is extraction temperature. Selection of an appropriate extraction temperature is crucial to minimise the oxidation of the olive oil sample inside the SPME vial, preventing the release of unwanted oxidation components [20]. More specifically, in unheated or heated-at-a-lower-temperature $\left(50^{\circ} \mathrm{C}\right)$ olive oil samples, a higher content of alcohols, esters, and terpenes has been observed compared to oils heated at higher temperatures; in the latter, higher amounts of aldehydes and carboxylic acids were found [21]. During oxidation off-flavor compounds produced due to fatty acid deterioration can be detected [22]. Consequently, the initially pleasant sensory characteristics of the oil attributed to compounds derived from the lipoxygenase (LOX) pathway eventually give way to unpleasant sensory attributes due to volatile compound deterioration. The screening of this parameter was performed by monitoring the total peak area of the selected components that are indicative of olive oil oxidation, while also monitoring components that are generally desirable according to the literature [21-24]. The selected compounds that indicate increased oil oxidation and must be kept at a minimum during sample heating were heptanal, 2-heptenal, octanal, and 2,4-heptadienal. The compounds that were selected as desirable for the analysis and contribute to its green odour notes [25] were 3-hexenal, (2E)-hexenal, 2-hexene-1-ol, hexan-1-ol, and 1-penten-3-one.

The study of extraction temperature showed an increase for both the desirable and undesirable oxidation components (Figure 1c). As indicated, there is a steep increase for the desirable components maximizing at around $55^{\circ} \mathrm{C}$, while the increase for the oxidation components appears to take place also around $55^{\circ} \mathrm{C}$, showing continuous increasing 
trend for the whole tested temperature range. This is in accordance with other related studies $[13,21]$, where extraction temperatures above $55^{\circ} \mathrm{C}$ were not considered and were not studied as a means to avoid thermal volatile alterations and oxidative degradation of the olive oil.

Regarding DOE-RSM optimization, a quadratic model including all linear terms, twoway interaction terms and second-order terms was fitted to the response data. The Multiple R-squared, Adjusted R-squared, $p$-value, and output of the Analysis of Variance (ANOVA) table for the model can be seen in Table 1.

Table 1. Model fit data along with corresponding output from the ANOVA analysis table.

\begin{tabular}{cccccc}
\hline Multiple R-Squared & 0.9028 & & & & \\
\hline $\begin{array}{c}\text { Adjusted R-Squared } \\
\boldsymbol{p} \text {-Value }\end{array}$ & $\begin{array}{c}0.7262 \\
4.02 \times 10^{-3}\end{array}$ & & & & \\
\hline & Df & Sum Sq & Mean Sq & F Value & Pr (>F) \\
\hline FO (x1, x2, x3, x4, x5) & 5 & $1.2055 \times 10^{18}$ & $2.4111 \times 10^{17}$ & 16.3748 & $9.02 \times 10^{-5}$ \\
TWI (x1, x2, x3, x4, x5) & 10 & $1.9382 \times 10^{17}$ & $1.9382 \times 10^{16}$ & 1.3164 & 0.3283 \\
PQ (x1, x2, x3, x4, x5) & 5 & $1.0584 \times 10^{17}$ & $2.1168 \times 10^{16}$ & 1.4376 & 0.2855 \\
Residuals & 11 & $1.6197 \times 10^{17}$ & $1.4724 \times 10^{16}$ & & \\
Lack of Fit & 6 & $4.5182 \times 10^{16}$ & $7.5303 \times 10^{15}$ & 0.3224 & 0.8996 \\
Pure Error & 5 & $1.1679 \times 10^{17}$ & $2.3357 \times 10^{16}$ & & \\
\hline
\end{tabular}

FO stands for First Order terms, TWI for Two Way Interaction terms and PQ for Pure Quadratic terms. Df are the Degrees of Freedom, Sum Sq the Sum of Squares and Mean Sq the Mean Square. Extraction temperature (x1), conditioning time (x2), extraction time (x3), desorption time (x4) and desorption temperature (x5).

As indicated by the Multiple R-squared, Adjusted R-squared, and $p$-value, the quadratic model is a good fit to the data and is statistically significant. There is also no evidence of lack of fit in the model. By studying Table S1, one can see that the most significant terms are extraction temperature and extraction time. The interaction between desorption temperature and desorption time also appears to be of significance.

A contour plot for the response surface of extraction time and extraction temperature can be seen in Figure 2. The plot indicates that the optimal point for these two factors is close to their maximum values. It also indicates that extraction time matters less as extraction temperature increases and vice-versa. For example, at a temperature of $85^{\circ} \mathrm{C}$, the same effect is achieved with an extraction time between 40 and $65 \mathrm{~min}$. However, the initial screening study indicated that at temperatures above $55^{\circ} \mathrm{C}$ an increase in oxidation products is witnessed. Therefore, a safer factor level selection would be an extraction temperature of $55{ }^{\circ} \mathrm{C}$ and an extraction time of $50 \mathrm{~min}$.

As the interaction between desorption time and desorption temperature appears significant, by studying their contour plot at Figure S1 at the selected extraction time and temperature, one can see a saddle-like plot, which suggests that optima lie with one factor maxed or the other. To keep desorption temperature as low as possible to avoid any oxidation or thermal degradation effects at the inlet, a selection of desorption temperature of $240{ }^{\circ} \mathrm{C}$ and desorption time of $12 \mathrm{~min}$ is made.

Regarding conditioning time, all the models' terms for this factor appear not to be significant, so a choice of the shortest time is made, in this case $5 \mathrm{~min}$. In summary, the SPME conditions chosen for analysis are $55{ }^{\circ} \mathrm{C}$ of extraction temperature, 5 min of conditioning time, $50 \mathrm{~min}$ of extraction time, and $240^{\circ} \mathrm{C}$ and $12 \mathrm{~min}$ desorption temperature and time, respectively.

The aim of the present study was the development of a method for the comprehensive profiling of the volatile content of VOOs. The oven program seemed to be appropriate for the most volatile components, since at $17 \mathrm{~min}$ and $180^{\circ} \mathrm{C}$ all components of interest were eluted. The DVB/CAR/PDMS fiber showed high extraction efficiency and was selected for further study. Extraction temperature and extraction time had a great effect on the detection of volatile components and their composition. With an extraction temperature of $55^{\circ} \mathrm{C}$ and an extraction time of $50 \mathrm{~min}$, an increased area of the total ion chromatogram was 
observed, preventing thermal oxidation or degradation of the compounds of interest. The optimum HS-SPME/GC-MS conditions were applied in the profiling method, facilitating the identification and semi-quantitation of 92 VOCs of different chemical classes, as shown in Table 2. A representative gas chromatogram of a Quality Control (QC) sample analysed with the optimal HS-SPME/GC-MS conditions is shown in Figure 3.

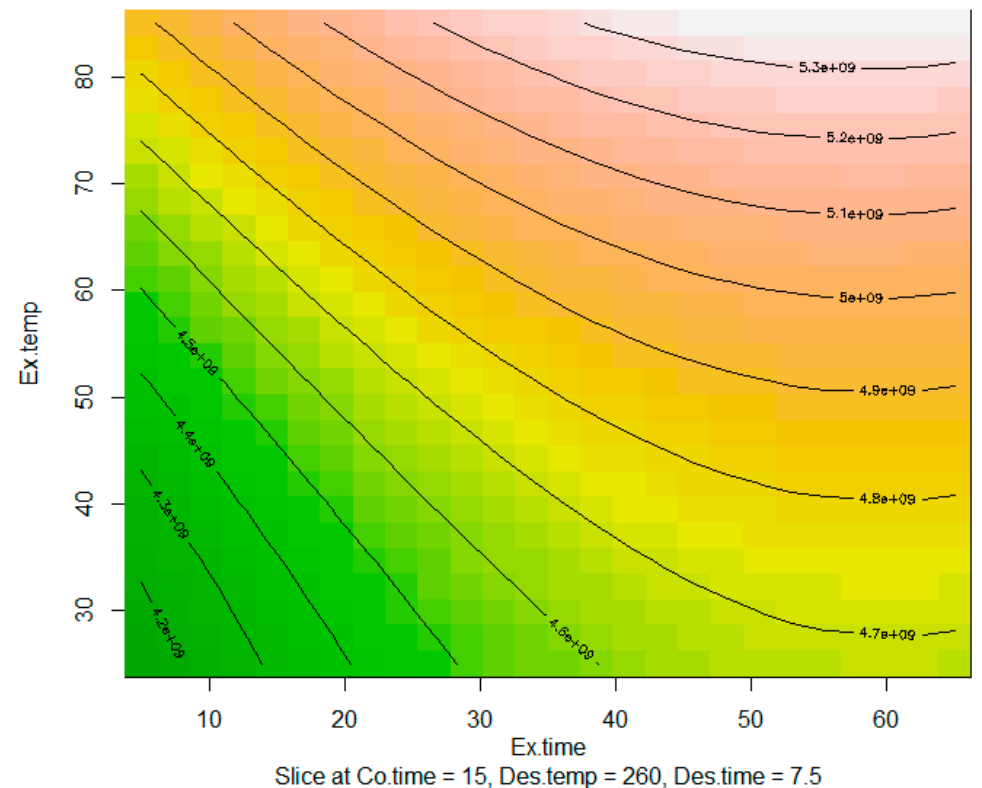

Figure 2. Contour plot for factors extraction temperature (Ex.temp) and extraction time (Ex.time). Green colour indicates lower response values and pink colour higher response values. Contours include corresponding response levels. The contour plot is a slice at the centre points for the rest of the remaining factors (Conditioning time (Co.time), Desorption temperature (Des.temp), and Desorption time (Des.time)). $\left(\mathrm{e}+08=\times 10^{8}\right)$.

Table 2. Chromatographic data (retention time, retention index) and mean values $(\mathrm{mg} / \mathrm{kg}$ ) relative to internal standard (Eucalyptol) and standard deviation for identified volatile compounds in Cretan EVOO samples.

\begin{tabular}{|c|c|c|c|c|c|c|c|c|c|c|c|}
\hline Compound Name & $\mathbf{R t}$ & RIexp & $\begin{array}{l}\text { RI } \\
\text { Lit }\end{array}$ & $\begin{array}{l}\text { Chania } \\
\text { Mean } \\
(n=17)\end{array}$ & $\pm \mathrm{SD}$ & $\begin{array}{c}\text { Heraklion } \\
\text { Mean } \\
(n=26)\end{array}$ & $\pm \mathrm{SD}$ & $\begin{array}{l}\text { Lasithi } \\
\text { Mean } \\
(\mathrm{n}=10)\end{array}$ & $\pm \mathrm{SD}$ & $\begin{array}{c}\text { Rethymnon } \\
\text { Mean } \\
(\mathrm{n}=10)\end{array}$ & $\pm \mathbf{S D}$ \\
\hline \multicolumn{12}{|l|}{ Alcohols } \\
\hline Isobutanol & 2 & 626 & 622 & 0.06 & 0.05 & 0.06 & 0.05 & 0.1 & 0.04 & 0.07 & 0.06 \\
\hline 1-Penten-3-ol, C5 & 2.54 & 683 & 686 & 1.96 & 0.38 & 1.69 & 0.49 & 1.45 & 0.49 & 1.05 & 0.37 \\
\hline 3-Methylbutan-1-ol & 3.33 & 738 & 744 & 0.12 & 0.05 & 0.14 & 0.05 & 0.15 & 0.05 & 0.13 & 0.07 \\
\hline 1-Pentanol & 3.89 & 771 & 766 & 0.09 & 0.08 & 0.08 & 0.06 & 0.04 & 0.05 & 0.17 & 0.05 \\
\hline (Z)-2-Penten-1-ol, C5 & 3.92 & 774 & 767 & 1.39 & 0.25 & 1.05 & 0.36 & 0.86 & 0.35 & 0.85 & 0.19 \\
\hline $\begin{array}{c}\text { (Z)-Hex-3-en-1-ol, } \\
\text { C6-LnA }\end{array}$ & 5.53 & 857 & 858 & 0.12 & 0.04 & 0.12 & 0.05 & 0.09 & 0.05 & 0.19 & 0.06 \\
\hline $\begin{array}{c}\text { (E)-Hex-2-en-1-ol, } \\
\text { C6-LnA }\end{array}$ & 5.82 & 871 & 866 & 1.99 & 1.75 & 2.23 & 2.49 & 0.72 & 2.36 & 7.96 & 3.45 \\
\hline Hexan-1-ol, C6-LA & 5.9 & 875 & 862 & 1.77 & 0.9 & 1.62 & 0.88 & 0.99 & 0.9 & 4.31 & 1.12 \\
\hline 1-Octanol & 9.76 & 1076 & 1074 & 0.08 & 0.02 & 0.08 & 0.03 & 0.07 & 0.02 & 0.07 & 0.04 \\
\hline 1-Nonanol & 11.48 & 1176 & 1172 & 0.06 & 0.03 & 0.1 & 0.05 & 0.1 & 0.04 & 0.05 & 0.04 \\
\hline (Z)-11-Hexadecen-1-ol & 12.31 & 1228 & - & 0.05 & 0.01 & 0.05 & 0.02 & 0.05 & 0.02 & 0.03 & 0.02 \\
\hline
\end{tabular}


Table 2. Cont.

\begin{tabular}{|c|c|c|c|c|c|c|c|c|c|c|c|}
\hline Compound Name & $\mathbf{R t}$ & RIexp & $\begin{array}{l}\text { RI } \\
\text { Lit }\end{array}$ & $\begin{array}{c}\text { Chania } \\
\text { Mean } \\
(n=17)\end{array}$ & \pm SD & $\begin{array}{c}\text { Heraklion } \\
\text { Mean } \\
(\mathrm{n}=26)\end{array}$ & \pm SD & $\begin{array}{l}\text { Lasithi } \\
\text { Mean } \\
(\mathrm{n}=10)\end{array}$ & \pm SD & $\begin{array}{c}\text { Rethymnon } \\
\text { Mean } \\
(\mathrm{n}=10)\end{array}$ & $\pm \mathrm{SD}$ \\
\hline \multicolumn{12}{|l|}{ Aldehydes } \\
\hline 3-Methylbutanal & 2.26 & 652 & 650 & 0.04 & 0.03 & 0.04 & 0.04 & 0.05 & 0.03 & 0.05 & 0.03 \\
\hline (E)-2-Pentenal, C5 & 3.52 & 749 & 754 & 0.08 & 0.03 & 0.06 & 0.03 & 0.06 & 0.02 & 0.04 & 0.02 \\
\hline (E)-2-Pentenal, C5 & 3.69 & 760 & 754 & 0.3 & 0.08 & 0.25 & 0.09 & 0.22 & 0.09 & 0.17 & 0.07 \\
\hline Pentanal & 2.75 & 703 & 695 & 0.99 & 0.44 & 0.87 & 0.4 & 0.75 & 0.39 & 0.37 & 0.22 \\
\hline 3-Hexenal, C6-LnA & 4.48 & 806 & 795 & 2.85 & 2.78 & 1.39 & 1.85 & 0.83 & 1.66 & 1.84 & 0.87 \\
\hline Hexanal, C6-LA & 4.52 & 808 & 798 & 3.51 & 1.42 & 3.1 & 1.06 & 2.75 & 1.07 & 4.22 & 1.08 \\
\hline 2-Hexenal, C6-LnA & 5.47 & 854 & 854 & 0.19 & 0.05 & 0.16 & 0.05 & 0.15 & 0.05 & 0.22 & 0.07 \\
\hline (2E)-Hexenal, C6-LnA & 5.61 & 862 & 856 & 25.43 & 9.62 & 22.16 & 9.57 & 21.51 & 9.23 & 32.67 & 13.59 \\
\hline Heptanal & 6.6 & 908 & 903 & 0.19 & 0.07 & 0.21 & 0.07 & 0.17 & 0.06 & 0.12 & 0.06 \\
\hline 2,4 Hexadienal & 6.79 & 919 & 916 & 1.95 & 0.71 & 1.43 & 0.61 & 1.22 & 0.57 & 1.66 & 0.34 \\
\hline Benzeneacetaldehyde & 9.39 & 1055 & 1049 & 0.03 & 0.01 & 0.04 & 0.02 & 0.06 & 0.02 & 0.06 & 0.02 \\
\hline Nonanal & 10.38 & 1111 & 1099 & 0.98 & 0.47 & 1.14 & 0.54 & 1.17 & 0.5 & 0.45 & 0.47 \\
\hline \multicolumn{12}{|l|}{ Acids } \\
\hline Acetic acid & 1.72 & 596 & 595 & 0.56 & 0.56 & 0.52 & 0.56 & 0.34 & 0.49 & 0.57 & 0.89 \\
\hline 3-Methylbutanoic acid & 5.35 & 852 & 858 & 0.02 & 0.01 & 0.02 & 0.01 & 0.02 & 0.01 & 0.03 & 0.01 \\
\hline Hexanoic acid & 8.04 & 981 & 981 & 0.73 & 0.71 & 0.55 & 0.51 & 0.21 & 0.49 & 0.7 & 0.35 \\
\hline Heptanoic acid & 9.72 & 1074 & 1076 & 0.1 & 0.03 & 0.09 & 0.03 & 0.06 & 0.03 & 0.09 & 0.03 \\
\hline 2-Ethylhexanoic acid & 10.52 & 1118 & 1123 & 0.58 & 0.55 & 0.37 & 0.45 & 0 & 0.41 & 0.37 & 0.41 \\
\hline Octanoic acid & 11.36 & 1170 & 1177 & 0.14 & 0.06 & 0.13 & 0.05 & 0.06 & 0.05 & 0.14 & 0.04 \\
\hline Nonanoic acid & 12.92 & 1268 & 1270 & 0.25 & 0.1 & 0.25 & 0.1 & 0.1 & 0.09 & 0.26 & 0.09 \\
\hline \multicolumn{12}{|l|}{ Esters } \\
\hline Ethyl acetate & 1.89 & 615 & 614 & 0.22 & 0.12 & 0.27 & 0.13 & 0.29 & 0.12 & 0.22 & 0.16 \\
\hline 3-Methylbutyl acetate & 6.04 & 882 & 883 & 0.13 & 0.08 & 0.13 & 0.07 & 0.18 & 0.07 & 0 & 0.11 \\
\hline (Z)-Pent-2-en-1-yl acetate & 6.76 & 918 & 916 & 0.59 & 0.33 & 0.39 & 0.25 & 0.27 & 0.23 & 0.51 & 0.11 \\
\hline $\begin{array}{l}\text { (3Z)-Hex-3-en-1-yl } \\
\text { acetate, C6-LnA }\end{array}$ & 8.55 & 1009 & 1005 & 18.49 & 9.03 & 12.15 & 7.19 & 13.46 & 7.22 & 7.97 & 4.09 \\
\hline Hexyl acetate, C6-LA & 8.68 & 1016 & 1011 & 4.5 & 2.44 & 3.07 & 1.85 & 4.21 & 1.89 & 2.81 & 1.51 \\
\hline \multicolumn{12}{|l|}{ Ethers } \\
\hline 1-Methoxy-2-propanol & 2.36 & 654 & 650 & 0.08 & 0.03 & 0.11 & 0.08 & 0.1 & 0.07 & 0.08 & 0.03 \\
\hline 1-Methoxyhexane & 4.99 & 830 & 831 & 0 & 0 & 0 & 0.02 & 0 & 0.06 & 0 & 0 \\
\hline $\begin{array}{l}\text { (3Z)-1-Methoxy-3- } \\
\text { hexene }\end{array}$ & 5.05 & 838 & 832 & 0 & 0 & 0 & 0 & 0 & 0.08 & 0 & 0 \\
\hline \multicolumn{12}{|l|}{ Ketones } \\
\hline 1-Penten-3-one, C5 & 2.58 & 687 & 679 & 1.96 & 0.92 & 1.65 & 1.17 & 1.46 & 1.07 & 0.7 & 1.15 \\
\hline 3-Pentanone & 2.71 & 701 & 694 & 1.65 & 0.92 & 1.26 & 0.72 & 1.04 & 0.75 & 1.51 & 0.77 \\
\hline $\begin{array}{l}\text { 2,2-Dimethyl-3- } \\
\text { heptanone }\end{array}$ & 9.64 & 1070 & - & 5.07 & 5.21 & 2.07 & 3.54 & 0.85 & 3.14 & 3.22 & 1.13 \\
\hline \multicolumn{12}{|l|}{ Terpenic Compounds } \\
\hline .alpha.-Pinene & 7 & 930 & 932 & 0.01 & 0.04 & 0.02 & 0.07 & 0 & 0.06 & 0.18 & 0.03 \\
\hline beta.-Pinene & 8.05 & 983 & 980 & 0.07 & 0.26 & 0.07 & 0.31 & 0 & 0.27 & 0 & 0.15 \\
\hline 6-Methyl-5-hepten-2-one & 8.2 & 990 & 985 & 0.61 & 0.41 & 0.56 & 0.33 & 0.51 & 0.31 & 0.78 & 0.34 \\
\hline Alpha-Terpinene & 8.79 & 1018 & 1017 & 0 & 0 & 0 & 0.01 & 0 & 0.01 & 0.02 & 0 \\
\hline
\end{tabular}


Table 2. Cont.

\begin{tabular}{|c|c|c|c|c|c|c|c|c|c|c|c|}
\hline Compound Name & $\mathbf{R t}$ & RIexp & $\begin{array}{l}\text { RI } \\
\text { Lit }\end{array}$ & $\begin{array}{l}\text { Chania } \\
\text { Mean } \\
(n=17)\end{array}$ & $\pm \mathrm{SD}$ & $\begin{array}{l}\text { Heraklion } \\
\text { Mean } \\
(n=26)\end{array}$ & $\pm \mathrm{SD}$ & $\begin{array}{l}\text { Lasithi } \\
\text { Mean } \\
(n=10)\end{array}$ & $\pm \mathrm{SD}$ & $\begin{array}{c}\text { Rethymnon } \\
\text { Mean } \\
(\mathbf{n}=10)\end{array}$ & $\pm \mathrm{SD}$ \\
\hline 3-Carene & 8.79 & 1018 & 1018 & 0 & 0.01 & 0.01 & 0.01 & 0 & 0.01 & 0.02 & 0 \\
\hline (Z)-beta-Ocimene & 9.29 & 1050 & 1048 & 0.05 & 0.02 & 0.07 & 0.03 & 0.07 & 0.03 & 0.09 & 0.02 \\
\hline o-Cymene & 8.95 & 1031 & 1037 & 0.14 & 0.48 & 0.2 & 0.59 & 0 & 0.54 & 1.33 & 0 \\
\hline D-Limonene & 9.02 & 1035 & 1041 & 0.7 & 1.83 & 0.99 & 3.29 & 0.1 & 2.87 & 0.19 & 4.33 \\
\hline$(+)$-2-Carene & 9.04 & 1035 & 1031 & 0 & 0 & 0.06 & 0.23 & 0 & 0.23 & 0.92 & 0 \\
\hline Neral & 12.61 & 1247 & 1244 & 0 & 0.01 & 0 & 0.01 & 0 & 0.01 & 0 & 0.01 \\
\hline Citral & 13.07 & 1276 & 1276 & 0 & 0.01 & 0 & 0.02 & 0 & 0.02 & 0 & 0.02 \\
\hline (+)-Cyclosativene & 14.67 & 1384 & 1368 & 0 & 0 & 0.01 & 0.01 & 0 & 0.01 & 0.01 & 0.01 \\
\hline Copaene & 14.73 & 1388 & 1392 & 0.03 & 0.01 & 0.05 & 0.03 & 0.06 & 0.03 & 0.1 & 0.02 \\
\hline Eremophilene & 16.34 & 1505 & 1503 & 0.01 & 0.01 & 0.01 & 0.01 & 0.01 & 0.01 & 0.01 & 0.01 \\
\hline alpha-Farnesene & 16.39 & 1509 & 1509 & 0.05 & 0.03 & 0.06 & 0.03 & 0.05 & 0.03 & 0.05 & 0.07 \\
\hline Kessane & 16.94 & 1551 & 1530 & 0 & 0 & 0 & 0 & 0 & 0 & 0 & 0 \\
\hline Liguloxide & 17.07 & 1561 & 1533 & 0 & 0 & 0.01 & 0.01 & 0 & 0.01 & 0 & 0.01 \\
\hline \multicolumn{12}{|l|}{ Others } \\
\hline 2-Methylpentane & 1.62 & 588 & 573 & 0.04 & 0.03 & 0.04 & 0.02 & 0.03 & 0.02 & 0.03 & 0.01 \\
\hline 3-Methylpentane & 3.39 & 741 & 748 & 0.05 & 0.03 & 0.07 & 0.03 & 0.08 & 0.03 & 0.09 & 0.03 \\
\hline Toluene & 3.86 & 770 & 771 & 0.17 & 0.1 & 0.19 & 0.21 & 0.13 & 0.24 & 0.06 & 0.2 \\
\hline 1-Octene & 4.25 & 794 & 792 & 0.17 & 0.1 & 0.15 & 0.08 & 0.14 & 0.07 & 0.21 & 0.05 \\
\hline Octane & 4.42 & 803 & 800 & 1.64 & 0.78 & 2.13 & 1.08 & 2.28 & 1 & 3.86 & 0.79 \\
\hline Styrene & 6.36 & 898 & 899 & 0.04 & 0.04 & 0.04 & 0.06 & 0 & 0.06 & 0.09 & 0.11 \\
\hline o-Xylene & 6.36 & 898 & 899 & 0.04 & 0.05 & 0.06 & 0.07 & 0.06 & 0.07 & 0 & 0.1 \\
\hline $\begin{array}{l}\text { (Prop-2-en-1- } \\
\text { yl)cyclopentane }\end{array}$ & 6.31 & 895 & 898 & 0.31 & 0.06 & 0.27 & 0.07 & 0.23 & 0.08 & 0.22 & 0.06 \\
\hline Pentene dimer 1, C5 & 6.4 & 899 & - & 0.22 & 0.05 & 0.19 & 0.06 & 0.16 & 0.06 & 0.15 & 0.05 \\
\hline Pentene dimer 2, C5 & 7.19 & 940 & 947 & 1.65 & 0.41 & 1.41 & 0.45 & 1.21 & 0.47 & 0.98 & 0.33 \\
\hline Pentene dimer 3, C5 & 7.33 & 947 & 949 & 1.9 & 0.46 & 1.54 & 0.53 & 1.35 & 0.53 & 1.22 & 0.34 \\
\hline $\begin{array}{l}\text { 4,8-Dimethyl-1,7- } \\
\text { nonadiene }\end{array}$ & 7.38 & 949 & 998 & 0.01 & 0.02 & 0 & 0.01 & 0 & 0.01 & 0 & 0 \\
\hline Propylbenzene & 7.59 & 960 & 962 & 0 & 0 & 0 & 0 & 0 & 0 & 0 & 0.02 \\
\hline 2,2,6-Trimethyloctane & 7.63 & 961 & 964 & 0 & 0 & 0.01 & 0.03 & 0 & 0.02 & 0 & 0.15 \\
\hline $\begin{array}{l}\text { 2,2-Dimethyl-3- } \\
\text { heptanone }\end{array}$ & 7.69 & 965 & 965 & 0.78 & 0.62 & 0.42 & 0.43 & 0.23 & 0.39 & 0.46 & 0.22 \\
\hline 1-Ethyl-2-methylbenzene & 7.73 & 971 & 969 & 0.05 & 0.03 & 0.04 & 0.04 & 0.03 & 0.04 & 0.04 & 0.05 \\
\hline 5-Ethyl-2(5H)-furanone & 7.82 & 971 & 968 & 12.91 & 12.72 & 5.39 & 8.7 & 2.22 & 7.76 & 6.5 & 3.27 \\
\hline 2,2,4-Trimethylpentane & 8.2 & 990 & - & 0.15 & 0.5 & 0.05 & 0.34 & 0 & 0.38 & 0 & 0.08 \\
\hline Pentene dimer 4, C5 & 8.27 & 994 & - & 0.88 & 0.23 & 0.77 & 0.23 & 0.66 & 0.24 & 0.58 & 0.18 \\
\hline Pentene dimer 5, C5 & 8.34 & 998 & - & 3.26 & 0.87 & 2.7 & 0.92 & 2.38 & 0.94 & 2.14 & 0.63 \\
\hline 2-Propylfuran & 8.45 & 1004 & - & 0.12 & 0.05 & 0.1 & 0.04 & 0.08 & 0.04 & 0.13 & 0.04 \\
\hline 4-Ethyltoluene & 8.89 & 1029 & - & 0.02 & 0.01 & 0.02 & 0.01 & 0.01 & 0.01 & 0.02 & 0.01 \\
\hline $\begin{array}{c}\text { 2,2,4,4,6- } \\
\text { Pentamethylheptane }\end{array}$ & 8.93 & 1030 & - & 0 & 0 & 0 & 0.01 & 0 & 0.04 & 0 & 0.07 \\
\hline 5-Ethyl-2(5H)-furanone & 9.17 & 1044 & - & 0.64 & 0.66 & 0.27 & 0.44 & 0.12 & 0.39 & 0.31 & 0.14 \\
\hline 1-Phenyl-1-propanone & 9.42 & 1059 & & 0 & 0 & 0 & 0.01 & 0 & 0 & 0 & 0 \\
\hline 1,4-Diethylbenzene & 9.53 & 1063 & 1070 & 0 & 0 & 0 & 0.01 & 0 & 0.01 & 0 & 0 \\
\hline 1-Undecene & 10.07 & 1078 & 1082 & 0.04 & 0.01 & 0.03 & 0.02 & 0.03 & 0.02 & 0.02 & 0.02 \\
\hline
\end{tabular}


Table 2. Cont.

\begin{tabular}{|c|c|c|c|c|c|c|c|c|c|c|c|}
\hline Compound Name & Rt & RIexp & $\begin{array}{l}\text { RI } \\
\text { Lit }\end{array}$ & $\begin{array}{c}\text { Chania } \\
\text { Mean } \\
(\mathrm{n}=17)\end{array}$ & \pm SD & $\begin{array}{l}\text { Heraklion } \\
\text { Mean } \\
(\mathrm{n}=26)\end{array}$ & \pm SD & $\begin{array}{l}\text { Lasithi } \\
\text { Mean } \\
(\mathrm{n}=10)\end{array}$ & \pm SD & $\begin{array}{c}\text { Rethymnon } \\
\text { Mean } \\
(\mathrm{n}=10)\end{array}$ & $\pm \mathrm{SD}$ \\
\hline $\begin{array}{l}\text { (E)-4,8-Dimethyl-1,3,7- } \\
\text { nonatriene }\end{array}$ & 10.11 & 1096 & 1098 & 0.06 & 0.02 & 0.09 & 0.04 & 0.09 & 0.04 & 0.05 & 0.03 \\
\hline Methyl benzoate & 10.27 & 1104 & 1106 & 0.04 & 0.01 & 0.05 & 0.02 & 0.05 & 0.02 & 0.03 & 0.01 \\
\hline Naphthalene & 11.88 & 1201 & 1206 & 0.06 & 0.24 & 0 & 0.15 & 0.01 & 0.13 & 0 & 0 \\
\hline (E)-2-Dodecene & 11.96 & 1206 & 1206 & 1.78 & 0.32 & 2.14 & 0.64 & 2.02 & 0.67 & 1.05 & 0.58 \\
\hline 1-Dodecene & 12.96 & 1270 & 1265 & 0.04 & 0.02 & 0.04 & 0.02 & 0.03 & 0.02 & 0.07 & 0.02 \\
\hline Pentadecane & 16.28 & 1501 & 1500 & 0.01 & 0.01 & 0 & 0.01 & 0 & 0.01 & 0.02 & 0.01 \\
\hline 2,4-Di-tert-butylphenol & 16.44 & 1513 & 1517 & 0.02 & 0.01 & 0.02 & 0.01 & 0.01 & 0.01 & 0.01 & 0.01 \\
\hline Total & & & & 108.1 & & 81.42 & & 92.67 & & 85.77 & \\
\hline C5 & & & & 13.6 & & 11.3 & & 12.35 & & 12.06 & \\
\hline C6 & & & & 54.37 & & 42.93 & & 47.86 & & 42.74 & \\
\hline $\mathrm{C} 5+\mathrm{C} 6$ & & & & 67.97 & & 54.22 & & 60.21 & & 54.8 & \\
\hline
\end{tabular}

Rt: Retention Time, I.S.: Internal Standard, RIex: Retention Index experimental, RIlit: Retention Index literature, SD: Standard Deviation.

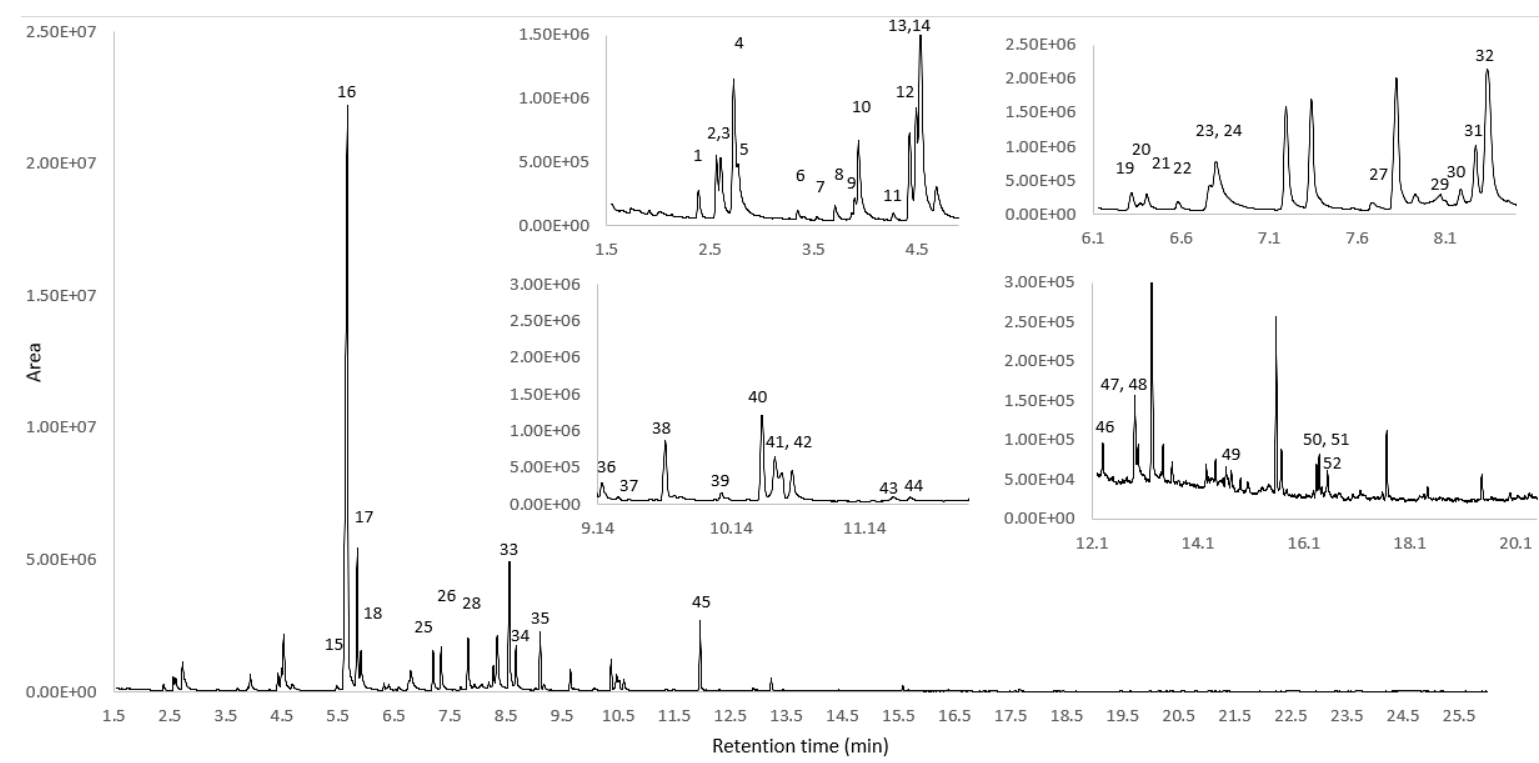

Figure 3. Major VOCs identified in the TIC of a QC olive oil sample analysed with optimised HS-SPME/GC-MS conditions. Upper in the insets, zoomed areas are shown. The identified components are given in numbers. (1) 1-methoxy-2-propanol, (2) 1-penten-3-ol, (3) 1-penten-3-one (4) 3-pentanone, (5) pentanal, (6) 3-methylbutan-1-ol, (7) (E)-2-pentenal, (8) (E)-2-pentenal, (9) 1-pentanol, (10) 2-penten-1-ol, (Z)-, (11) 1-octene, (12) octane (13) 3-hexenal, (14) hexanal, (15) (Z)-hex-3-en-1-ol, (16) (2E)-hexenal, (17) (E)-2-hexen-1-ol, (18) 1-hexanol, (19) (prop-2-en1-yl)cyclopentane, (20) o-xylene, (21) 3-ethyl-1,5-octadiene, (22) heptanal, (23) (Z)- 2-penten-1ol, acetate, (24) 2,4-hexadienal, (25) 3-ethyl-1,5-octadiene, (26) 3-ethyl-1,5-octadiene, (27) 2,2dimethyl-3-heptanone, (28) 5-ethyl-2(5H)-furanone, (29) hexanoic acid, (30) 6-methyl-5-hepten-2-one, (31) 3-ethyl-1,5-octadiene, (32) 3-ethyl-1,5-octadiene, (33) (3Z)-hex-3-en-1-yl acetate, (34) hexyl acetate, (35) eucalyptol (I.S), (36) benzyl alcohol, (37) o-cymene, (38) 2,2-dimethyl-3-heptanone, (39) 1-undecene, (40) nonanal, (41) (E)-4,8-dimethyl-1,3,7-nonatriene, (42) 2-ethylhexanoic acid, (43) octanoic acid, (44) 1-nonanol, (45) (E)-2-dodecene, (46) 11-hexadecen-1-ol, (Z)-, (47) nonanoic acid, (48) 1-dodecene, (49) copaene, (50) pentadecane, (51) eremophilene, (52) alpha-farnesene. $\left(\mathrm{E}+07=\times 10^{7}\right)$ 


\subsection{Identification of VOCs by HS-SPME/GC-MS Analysis}

Olive oil volatile compounds are primarily derived from the enzymatic oxidation of the fatty acids linoleic and linolenic. Positive aroma perceptions in olive oil are produced by endogenous plant enzymes, through the LOX, while aroma defects are linked with chemical oxidation and exogenous enzymes [5]. The volatile fraction of VOOs mainly consists of aldehydes, ketones, aliphatic and aromatic hydrocarbons, ethers, esters, aliphatic and triterpenic alcohols, and furan derivatives. Aroma compounds responsible for green desirable notes, found in high-quality VOOs, are produced enzymatically from polyunsaturated fatty acids through the LOX pathway. It has been observed that in the aroma of these oils, $\mathrm{C} 6$ aldehydes and alcohols and their corresponding esters are the most abundant accumulation products [26]. Both major and minor VOCs are important to olive oil aroma, since minor compounds with low odor threshold values are frequently considered significant contributors. Even those whose levels are below their olfactory threshold can still provide useful quality marker information [27].

In all samples, C6 and C5 VOCs derived from the LOX pathway were the most abundant (Figure $4 a, b$ ). This is in accordance with the organoleptic results obtained by the tasting panel, where samples were identified as EVOOs with medium fruitiness attributes $(3<x<6)$. C6 and C5 VOCs contribute to the delicate green aroma of VOO, which enhances the "green" fruitiness attribute and is highly valued by consumers. The formation of these compounds is mainly influenced by the cultivar, the degree of ripeness of the fruit, the storage period of fruits before oil extraction, and the processing method [28].

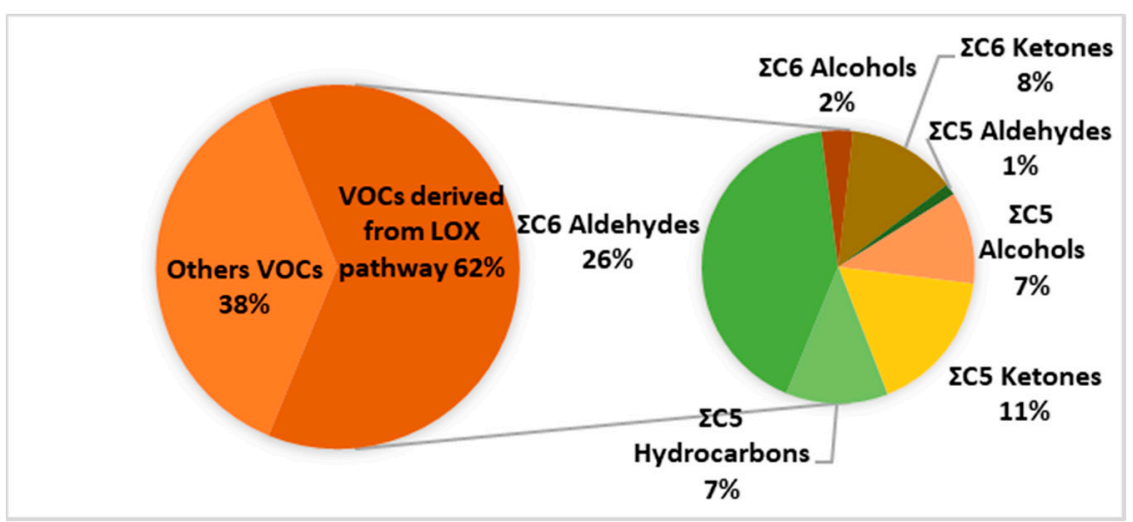

(a)

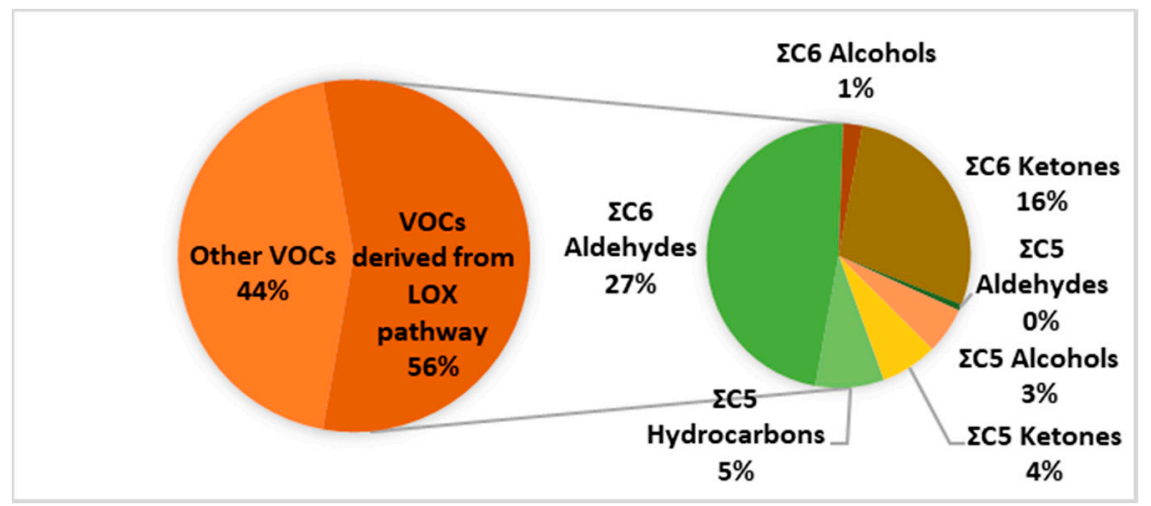

(b)

Figure 4. Natural variation of VOCs derived from LOX pathway in a (a) Koroneiki and (b) Tsounati variety sample.

C6 aldehydes produced from the linolenic acid, E-2-hexanal, 3-hexenal, and Z-2hexanal, were found in considerable amounts. In agreement with data reported in other 
studies examining European olive oil cultivars, (2E)-hexenal, responsible for the "green" attributes, was the most prevalent aroma compound in all VOO samples [20]. More specifically, concerning the Koroneiki cultivar, (2E)-hexenal ranged from $7.64 \mathrm{mg} \mathrm{kg}^{-1}$ in a conventional sample to $51.25 \mathrm{mg} \mathrm{kg}^{-1}$ in an organic sample from the Heraklion region unit. In a similar manner, Z-3-hexanal, which is positively correlated with fruity, leave/grass, and almond notes [29], was found in high levels in VOO samples, reaching $8.77 \mathrm{mg} \mathrm{kg}^{-1}$ in an organic sample from Chania. High amounts of hexanal, produced by breaking 13hydroperoxide from linoleic acid, are frequently related to sensory notes sweet, apple, and green [30]. In the studied samples, hexanal reached $8.04 \mathrm{mg} \mathrm{kg}^{-1}$ in an organic sample. C6 alcohols such as Z-3-hexen-1-ol, E-2-hexen-1-ol, and hexan-1-ol, related to sweetness, were also identified [5]. Z-3-hexen-1-ol and hexan-1-ol were then esterified by the alcohol acetyltransferase to produce the corresponding esters (3Z)-hex-3-en-1-yl acetate and hexyl acetate, which also were found in considerable amounts in the studied samples ranging from 2.13 to $40.08 \mathrm{mg} \mathrm{kg}^{-1}$ and from 0.5 to $9.75 \mathrm{mg} \mathrm{kg}^{-1}$, respectively. The contribution of these volatiles is quite significant, as they complement aromas with sweet and pleasant notes [31].

Aside from C6 molecules, the headspace of VOO contains a reasonable amount of C5 alcohols and C5 carbonyl compounds [32]. The existence of these VOCs indicates the development of a new branch of the LOX pathway that produces $\mathrm{C} 5$ compounds. This extra branch is active during the biogeneration of olive oil aroma. C5 aldehydes and alcohols contribute to the positive flavor attributes of olive oil, providing pungent sensations, and are correlated to bitterness [5]. The molecule 1-penten-3-ol is related to "mouldy" and "rancid" defects associated with lawn, olive, leaf, and pungency and ranged from $0.90 \mathrm{mg} \mathrm{kg}^{-1}$ in conventional VOO of Heraklion to $2.94 \mathrm{mg} \mathrm{kg}^{-1}$ in an organic sample of Koroneiki cultivar in Chania. (Z)-2-penten-1-ol (0.60-1.87 $\left.\mathrm{mg} \mathrm{kg}^{-1}\right)$ and 2-pentenal (E)- $\left(0.1-0.51 \mathrm{mg} \mathrm{kg}^{-1}\right)$ are minor compounds whose presence is correlated with banana, sweet, fruity and green, apple, and bitter almond attributes, respectively [33]. C5 ketones, pentene dimers, or monoterpenes affect olive oil aroma even in low concentrations. The molecule 1-penten-3one reached high amounts at the Koroneiki samples of Heraklion $\left(5.75 \mathrm{mg} \mathrm{kg}^{-1}\right)$ whereas it was not detected in an organic $\mathrm{VOO}$ of the Koroneiki variety from Rethymnon. Its presence is linked to leaf, mustard, and pungent notes [34], whereas higher amounts have been also related to metallic notes. The isomer 3-ethyl-1,5-octadiene is produced over the oil extraction process through the same branch of the LOX pathway as the C5 compounds and was detected in all samples (0.12-4.91\%) [35]. Other minor volatiles, terpene hydrocarbons (mono- and sesquiterpenes), were detected in Cretan VOOs, and despite the fact they do not contribute to VOO aroma due to their low concentration and high odor threshold, they constitute significant quality markers [29]. Among all terpenoid compounds, the most abundant in the studied VOO samples, were d-limonene $\left(0.04-20.18 \mathrm{mg} \mathrm{kg}^{-1}\right)$ with citrus, mint odor perceptions, $\alpha$-farnesene $\left(0.02-0.27 \mathrm{mg} \mathrm{kg}^{-1}\right)$ with floral, herb, wood, and sweet odor qualities [36], and (E)-4,8-dimethyl-1,3,7-nonatriene $\left(0.04-0.19 \mathrm{mg} \mathrm{kg}^{-1}\right)$. Other terpenoid volatiles such as alpha- and beta-pinene, o-cymene, and copaene were also detected in lower amounts. Some rare terpenic compounds such as citral, (+)-cyclosativene, eremophilene, neral, 3-carene, and kessane, which so far have been reported only in a few studies $[29,37,38]$, were also identified with the current method.

The detection of benzenes, toluene, xylenes, and styrene (BTEXS) in olive oil is of high importance. These VOCs are widely distributed in the environment and food products mainly due to emissions from vehicles, bonfires, and paints into the ambient air near orchards. In olive oil, BTEXS are frequently identified, and their presence is attributed to several factors, such as biological processes in the fruit, the production technology used, contamination by fuel vapors, etc. [39]. In this study, toluene, styrene, xylene, propylbenzene, 1-ethyl-2-methylbenzene, 4-ethyltoluene, 1,4-diethylbenzene, and naphthalene were identified. The presence of styrene in VOO samples has been attributed to natural causes, such as decarboxylation of the cinnamic acid that is naturally present in the olive pulp or by migration from the plastic package [39]. Naphthalene is usually hanged on trees 
by olive farmers, as it has a characteristic aroma that is theorised to repel the olive fly (Bactrocera oleae), and in this study was identified in low levels $\left(0.01-0.92 \mathrm{mg} \mathrm{kg}^{-1}\right)$ in 8 out of 63 samples [40].

According to our knowledge, VOCs such as 1-propanone-1-phenyl, benzene, 1,4diethyl, 11-hexadecen-1-ol, (Z), and 2,4-di-tert-butylphenol are reported for the first time in VOO samples.

\subsection{Classification of Cretan VOO Samples}

EVOO flavor is considered a significant quality but may also be utilized as a classification criterion. VOO volatile profile plays a fundamental role in defining the geographical origin of oils. The majority (roughly 70\%) of PDO/PGI olive oils in Greece are produced in the Peloponnese and Crete. Six of the ten Cretan PDOs come exclusively from Koroneiki, the main Greek cultivar and also the most prominent in Crete; three include blends of $90 \%$ Koroneiki VOO, and only one contains blends of $60 \%$ oil from Tsounati, the second most significant Cretan cultivar [41]. One PDO was registered for the entire Lasithi regional unit, whilst two PDOs from Chania come from two distinct terroirs but include comparable blends of Koroneiki and Tsounati oils. It is essential that these specific PDOs be subjected to thorough administrative control. These objective criteria, including organoleptic ones, should be accessible to support the authenticity and prevent attempts at fraud.

In the current study, the potential of the newly developed method was investigated for its use in the classification of Cretan VOO samples with the aid of multivariate statistical analysis. The obtained data were analysed using multivariate statistical analysis models to identify correlations between VOC profiles and VOO geographical origin; thus, chromatographic peak areas were used for modelling the differences between the studied groups. The overall performance of the analytical method was evaluated by assessing the variability of the detected compound in QC samples. All the compounds in QC samples presented \% RSD $<30 \%$, indicating satisfactory stability and reproducibility of the analytical system during the analytical batch. As shown in Figure S2, principal component analysis (PCA) was used to evaluate the analytical system's suitability and reproducibility (QC cluster). Group differentiation and identification of potential markers related to geographical origin were performed by latent structures OPLS DA analysis.

Before the assessment of geographical origin to the volatile fingerprint of VOOs, the effect of farming type (organic vs. conventional) was examined. Multivariate statistical analysis was performed to samples from each area produced with different cultivation types. It was observed that farming type is not a statistically significant factor for the specific samples, and thus geographical origin assessment was further performed using both sample types (organic and conventional). Initially, VOO samples from nearby production regions such as Chania-Rethymnon, Rethymnon-Heraklion and Lasithi-Heraklion (Figure S3) did not present any statistically significant differences regarding their volatile content. Although the region of Chania is located on the western side of Crete and Heraklion roughly in the middle of the island, the content of VOOs was not affected by the different origins due probably to other stronger common factors that may affect the VOO profile, which may include climate, fruit maturity, and processing technology [5]. The impact of geographical origin was found significant when VOOs from Lasithi, the easternmost region of Crete, were compared with samples from Chania and Rethymnon. The respective orthogonal projection to latent structures discriminant analysis (OPLS-DA) models are illustrated in Figure 5a,b, where samples with the same origin are clustered together and show clear differentiation against samples from Lasithi. Clear separation was observed between VOOs from Chania and Lasithi and between VOOs from Lasithi and Rethymnon with cross-validated ANOVA analysis (CV Analysis), $p=0.0008$ and $p=0.02$, respectively. Permutation tests showed that the models were robust, with high predictability (R2Y (cum) and Q2 values of 0.827 and 0.656 and 0.881 and 0.453 , respectively). Studying the multivariate loadings vectors in each case revealed various analytes related to the differentiation between the studied groups. As a final step, the unpaired Student's t-test was performed to 
establish and confirm the significance of important metabolites. All statistically significant metabolites and their variations in each group are presented as box plots in Figure 6 .

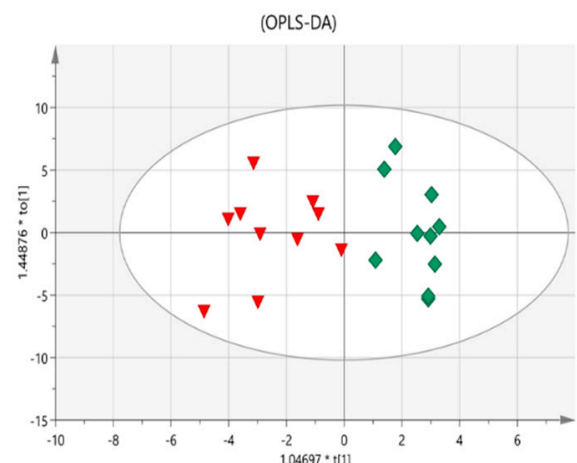

(a)
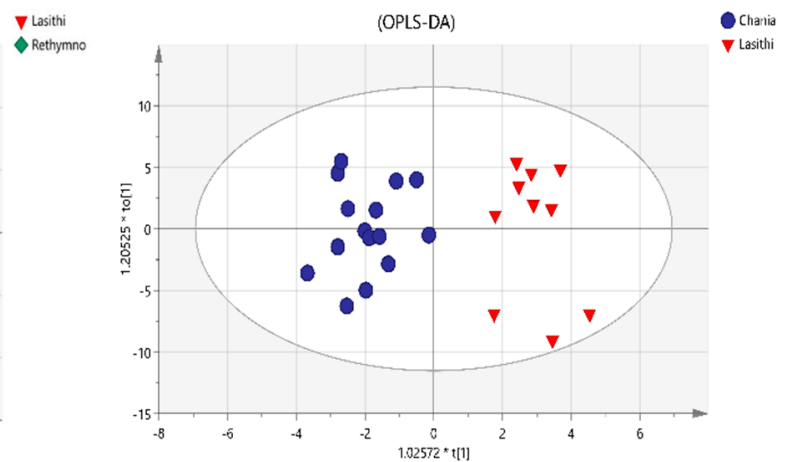

(b)

Figure 5. Orthogonal Projections to Latent Structures Discriminant Analysis (OPLS-DA) on EVOO samples volatile profile, using the 'geographical origin' as class membership criterion, (a) Lasithi vs. Rethymno, (b) Chania vs. Lasithi.

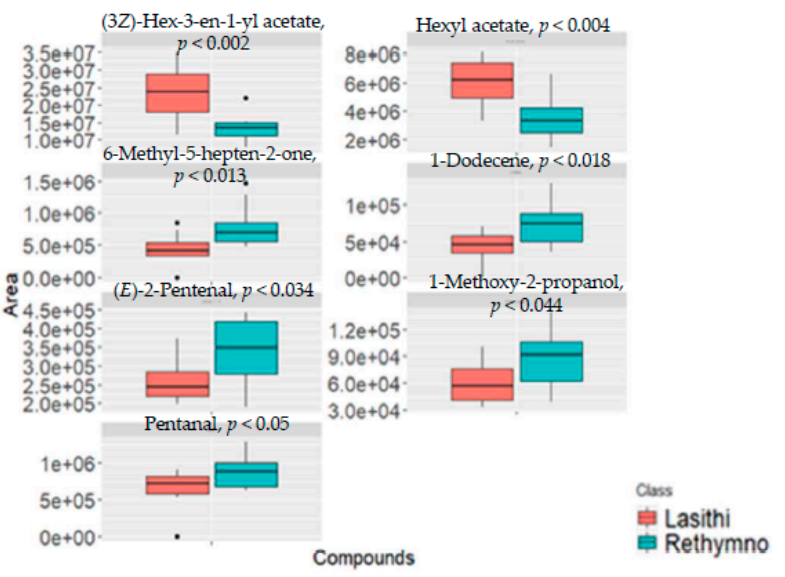

(a)

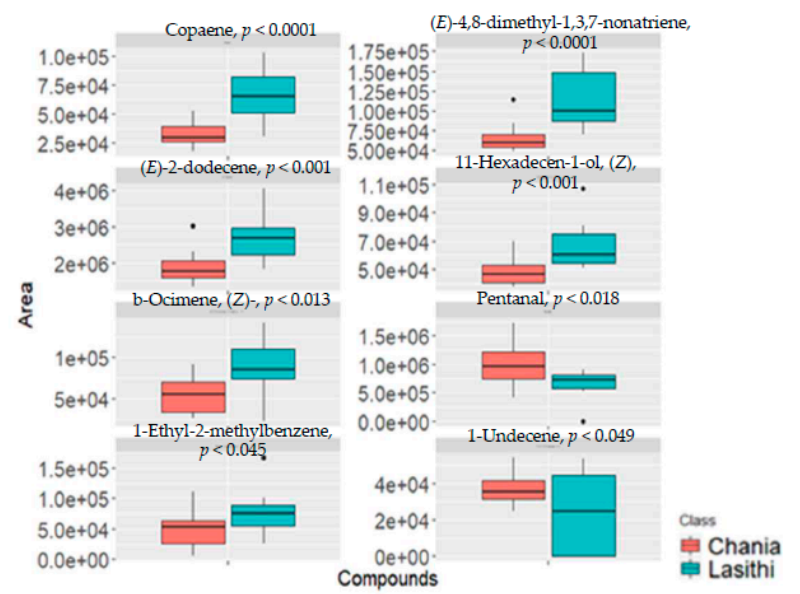

(b)

Figure 6. Box plots of identified biomarkers in VOOs samples. Boxes are drawn from the 25th to 75th percentiles in the concentration distribution. (a) Lasithi vs. Rethymnon, (b) Chania vs. Lasithi. $\left(\mathrm{e}+07=\times 10^{7}\right)$. 
The compounds found significant $(p<0.05)$ for geographical differentiation consisted of four terpenic hydrocarbons (6-methyl-5-hepten-2-one, copaene, $(E)-4,8$-dimethyl1,3,7-nonatriene, and (Z)-beta-ocimene), two esters ((3Z)-hex-3-en-1-yl acetate and hexyl acetate), two aldehydes ((E)-2-pentenal and pentanal), three hydrocarbons (dodecene, $(E)$ 2-dodecene, undecene, and 1-ethyl-2-methylbenzene), one alcohol ((Z)-11-hexadecen-1-ol), and one ether (1-methoxy-2-propanol), as shown in Figure 6. Eight of them, 6-methyl-5hepten-2-one, copaene, (E)-4,8-dimethyl-1,3,7-nonatriene, (Z)-beta-ocimene, (3Z)-hex-3-en1-yl acetate, hexyl acetate, (E)-2-pentenal, and dodecene, have previously been reported as markers for the classification of Greek VOO according to geographical origin [42,43]. More specifically, efforts have been put on studies that focus on the differentiation of olive oils produced in different islands of north-western Greece [43] (Zakynthos, Kefalonia, Lefkada, Kerkyra, Preveza, and Messologi) and different regions [42] (Messinia and Lakonia in the Peloponnese, Irakleio on the island of Crete, and Etoloakarnania in central Greece).

In this study, when samples from Lasithi and Rethymnon were compared, seven volatiles were found to be responsible for the differentiation between the groups. Three of them ((3Z)-hex-3-en-1-yl acetate, hexyl acetate and (E)-2-pentenal) are produced through the LOX-pathway, contributing to olive oil aroma. The volatiles 1-methoxy-2-propanol and 1-dodecene have also been identified as potential markers in Croatian monovarietal/PDO EVOO [44], while pentanal has shown high discrimination ability for the classification of north Moroccan olive oils [45]. A ripening indicator with fruity-like odor, 6-Methyl-5hepten-2-one, has been detected as a marker for the characterisation of Turkish olive oils according to origin (Mediterranean, Aegean, South-eastern Anatolia, Marmara, and Black Sea) [46]. Overall, (3Z)-hex-3-en-1-yl acetate, and hexyl acetate were found upregulated in samples from Lasithi while all the others were increased in samples from Rethymnon. Pentanal was found as statistically significant in both origin studies of VOOs. Samples from Lasithi showed lower levels of pentanal compared to Rethymnon and Chania.

In total, eight compounds were found to differ between VOOs from Chania and Lasithi. Three terpenic hydrocarbons, copaene, with a sweet-fruity description, $(E)$-4,8-dimethyl1,3,7-nonatriene, and (Z)-beta-ocimene, with an herb aroma description, were among the most important discriminant volatiles. This is in accordance with several studies where terpene hydrocarbons were reported as suitable markers of the geographic origin and genotype of EVOOs [29,47,48]. (E)-2-Dodecene has been reported as a discriminant variable in a study investigating VOOs of Chemlali and Neb Jmel cultivars growing in central and south Tunisia [49]. In this study, 1-ethyl-2-methylbenzene, 1-undecene, and (Z)-11hexadecen-1-ol are reported as discriminant volatiles for geographical origin classification for the first time. In general, pentanal and 1-undecene, were found in increased amounts in oils from Chania, while the other statistically significant compounds were found in decreased levels in oils from Lasithi.

The aroma profile of the monovarietal Koroneiki VOOs examined was mainly characterized by the presence of C6 LOX alcohols, aldehydes, and esters as well as the contribution of C5 LOX compounds similarly to literature reports for VOOs of the same cultivar [6,50]. VOCs concentrations in the monovarietal Koroneiki VOOs of the current study varied significantly either as total content, or as total group content, or even as content of most individual VOCs. Differences were considered significant even within the same farming type (organic or conventional) or production region, revealing the complexity of the VOO aroma profile. The latter is in accordance with reported findings on variable profiles expected due to the effect and synergism of various biotic and abiotic parameters, such as agricultural practices, altitude, harvest time, and conditions employed from harvest-to-oil production and storage [51]. Nonetheless, VOCs identified in the current study as positive contributors (e.g., (2E)-hexenal, hexanal, hexan-1-ol, 1-penten-3-one, hexyl acetate, and hexenyl acetate), in terms of amount and/or importance of contribution to overall profile (relevant to OTV), have been also recognized as such for monovarietal Koroneiki VOOs studied [6,50]. Moreover, terpenic compounds useful indicators for geographical and botanical VOO differentiation have been reported to be present in the aroma profile of 
Koroneiki VOOs [6,50], which is in line with current findings; limonene, copaene, and a farnesene were present in all monovarietal Koroneiki VOO samples studied.

The application potential of the method could be improved by analysing a larger dataset of EVOO samples and/or samples from oncoming harvest years from the same areas. We are planning to expand our data set in this direction, analysing samples produced in subsequent harvest years. In addition, we plan to use this technology to compare Cretan vs. non-Cretan VOOs, a comparison that could be of legislating and commercial significance.

\section{Materials and Methods}

\subsection{Samples}

A total of 63 bottled and branded VOO samples that were previously evaluated for quality parameters were collected for the 5 th Cretan olive oil competition. The samples were produced from olives of both organic $(n=19)$ and conventional $(n=44)$ olive groves and different local olive cultivars (Koroneiki and Tsounati) harvested in 2018-2019 in different regions of Crete (Chania $\mathrm{n}=15$, Rethymnon $\mathrm{n}=12$, Lasithi $\mathrm{n}=10$, and Heraklion $\mathrm{n}=26$ ) (Table S2). The organoleptic assessment of the olive oil samples was performed according to the Official EU method EEC/2568/91 [4] and its amendments by the trained sensory panel of the accredited Sensory Evaluation Laboratory of Crete ACR in Rethymnon Crete, Greece, and stored at $-20^{\circ} \mathrm{C}$ in dark glass vials that were shielded from light and oxygen.

\subsection{Chemicals}

LC-MS grade methanol was purchased from Sigma-Aldrich (Darmstadt, Germany). The internal standard eucalyptol (1,8-cineole) was of analytical grade and was purchased from Sigma-Aldrich (Darmstadt, Germany).

\subsection{Optimisation of SPME Extraction Conditions}

Initially, the effect of 3 experimental conditions for the HS-SPME method was studied by triplicate analysis following the well-established one-factor-at-a-time approach (OFAT). Sample mass was studied in the range of (1.000-4.000 g) in order to investigate any fiber saturation effects. Additionally, a range of settings was investigated for stirring of the sample (100-300 rpm). The latter two were optimised using the total peak area. The effects of extraction temperature for VOCs that are both desirable and undesirable were screened in the range of 30 to $85^{\circ} \mathrm{C}$ so that an initial assessment was made for this crucial experimental parameter. Regarding the optimisation of the rest SPME conditions, a modelling approach was performed using the DOE and RSM approaches for the total peak area of each chromatogram representing all the ionisable and detectable volatile components of the sample. The optimisation was performed using a base fractional factorial (FF) $2^{5-1}$ design with 5 factors (resolution $\mathrm{V}$ ) that was augmented to a circumscribed central composite (CCC) with an alpha value of 2 . A total of 32 runs included 16 base runs for FF (cube portion), 6 centre points to measure the experimental error, and 10 runs for CCC (star portion). Runs were randomised for each portion and were run in a single analytical sequence. The SPME factors selected for optimisation were extraction temperature, conditioning time, extraction time, desorption temperature, and desorption time. Although a preliminary screened factor, extraction temperature was included in the model as a significant factor to have a more complete picture of the SPME condition effects and to increase the model's applicability. The experimental area for the corresponding factors is given in Table 3, with the corresponding design provided in Table S3. Factor and level selection was made based upon instrumental limitations, fit-for-purpose needs, and by referring to relevant literature $[18,52]$.

The response selected for optimisation was the total chromatogram area, as measured by the sum of the areas of each ion, after the removal of the ions that were attributed mainly to siloxane contamination, as described in Section 3.6. 
Table 3. Factors and their corresponding levels in each portion of the RSM design: cube portion for the FF base design and star portion for the CCC augmentation.

\begin{tabular}{cccccc}
\hline Abbr. & Cube Level 1 & Cube Level 2 & Star Level 1 & Star Level 2 & Units \\
\hline Ex.temp & 40 & 70 & 25 & 85 & ${ }^{\circ} \mathrm{C}$ \\
Co.time & 10 & 20 & 5 & 25 & mins \\
Ex.time & 20 & 50 & 5 & 65 & mins \\
Des.temp & 250 & 270 & 240 & 280 & ${ }^{\circ} \mathrm{C}$ \\
Des.time & 5 & 10 & 2.5 & 12.5 & mins
\end{tabular}

Ex.temp stands for extraction temperature, Co.time for conditioning time, Ex.time for extraction time, Des.temp for desorption temperature, and Des.time for desorption time. Units are standard SI units for each factor.

\subsection{Sample Preparation}

The optimal SPME conditions were set following the approach described in Section 3.3. Briefly, 1.85 gr of olive oil were weighed into a $15 \mathrm{~mL}$ glass vial, and $4 \mu \mathrm{L}$ of a $1000 \mu \mathrm{g} / \mathrm{mL}$ eucalyptol internal standard solution in methanol were added, reaching a final concentration of $2 \mu \mathrm{g} / \mathrm{mL}$. The vials were closed with a PTFE/silicone septum and equilibration was performed with a PAL Shimadzu autosampler unit (AOC 6000, CTC Analytics, Zwingen, Switzerland) at $55 \pm 0.1{ }^{\circ} \mathrm{C}$ for $15 \mathrm{~min}$ upon agitation at $250 \mathrm{rpm}$. The (DVB/CAR/PDMS) fiber ( $2 \mathrm{~cm}$ length, $50 / 30$ thickness) (Sigma-Aldrich) was then introduced to the headspace for $50 \mathrm{~min}$ at $55^{\circ} \mathrm{C}$. The fiber was pre- and post-conditioned for $10 \mathrm{~min}$ at $260^{\circ} \mathrm{C}$, according to the manufacturer's instructions.

\subsection{Gas Chromatography-Mass Spectrometry}

VOCs were analyzed using a Shimadzu GCMS-QP2020 instrument equipped with a PAL SHIMADZU autosampler unit (AOC 6000, CTC Analytics, Zwingen, Switzerland). Chromatographic separation was performed on a MEGA-5 MS capillary column $(30 \mathrm{~m} \times 0.25 \mathrm{~mm}, 0.25 \mu \mathrm{m})($ MEGA, Legano, Italy). Injection was operated in split mode (1:2) at $250{ }^{\circ} \mathrm{C}$; He as the carrier gas at a flow rate of $1.2 \mathrm{~mL} / \mathrm{min}$; the column was held for $2.5 \mathrm{~min}$ at $40{ }^{\circ} \mathrm{C}$, then programmed at $10^{\circ} \mathrm{C} \mathrm{min}-1$ to $230{ }^{\circ} \mathrm{C}$ and held for $5 \mathrm{~min}$. The mass detector was operated in the electron impact mode at $70 \mathrm{eV}$. Temperatures of MS source and quadrupole were set to 240 and $200{ }^{\circ} \mathrm{C}$, respectively. The mass spectra scanned at $\mathrm{m} / \mathrm{z}$ 40-450 amu range.

A pooled sample $(\mathrm{QC})$ was prepared as a representative sample by mixing equal volumes of each VOO sample. The QC sample was injected at the start, every seven samples, and the end of the run to evaluate instrument stability. Blank runs were performed during the study to reveal possible carryover. The samples were analysed in randomised order.

\subsection{Data Processing and Chemometrics}

The Shimadzu software LabSolutions, GCMS Solutions version 2.50 SU3LabSolution, was used for acquiring the GC-MS data. Assignment Validator and Integrator (GAVIN) script for MATlab was used for performing peak integration complementary to Automated Mass spectral Deconvolution and Identification System (AMDIS) for peak deconvolution and identification. Total peaks areas for RSM-related modeling were extracted from the raw chromatogram files using OpenChrom ${ }^{\circledR}$ Lablicate Edition version 1.4 (Lablicate $\mathrm{GmbH}$, Hamburg, Germany) [53] including the area of all scanned ions minus those attributed to siloxane contamination from column: 207, 281, and 355 [54]. Compound identification was performed by comparing mass spectra of eluting compounds with those of commercial libraries (NIST17 and FFNSC3) and by calculating linear retention indices relative to a series of n-alkanes (C8-C24) (Sigma-Aldrich). The VOCs were semi-quantified by dividing the peak areas of the compounds of interest by the peak area of the internal standard (Eucalyptol) and multiplying this ratio by the initial concentration of the internal standard (expressed as $\mathrm{mg} \mathrm{kg}^{-1}$ ). The peak areas were measured from the full scan chromatograph using total ion current (TIC). 
DOE design and analysis were performed using $R$ version 3.6.2 [55] with RStudio (RStudio, Inc., Boston, MA, USA) [56] and DOE/RSM-related functionality [57-59]. The SIMCA package (version 13.0.2.; Umetrics, Sweden) was used for multivariate statistical analysis and biomarker assessment via VIP plots (Variable Importance for the Projection), loading plots, S-plots, p (corr), and hotelling's lines. Principal components analysis (PCA) and orthogonal projection to latent structures discriminant analysis (OPLS-DA) were performed to assess data in a multivariate setting. Models' validation was evaluated using permutation plots and CV-ANOVA value. Two-tailed t-test, with unequal variance and a threshold of $p<0.05$, and ANOVA were employed in Microsoft Excel Spreadsheets.

\section{Conclusions}

In this study, a HS-SPME/GC-MS method by Design of Experiments (DoE) and Response Surface Methodology (RSM) approaches was successfully optimized. The optimized parameters were sample amount, sample stirring, extraction temperature, conditioning time, extraction time, desorption temperature, and desorption time. The optimized HSSPME/GC-MS method allowed the identification and semi-quantification of a high number of compounds. Of the 92 VOCs identified, 4 are found for the first time in EVOO while others, mainly terpenoid compounds, are rarely identified in EVOO, with the use of SPME/GC-MS. Statistical analysis allowed for identifying markers with significance in the geographical classification of Cretan EVOO samples. The method is deemed useful for the analysis of EVOO, providing easy, automated, efficient, and economic sample preparation and analysis along with information-rich GC-MS data.

Supplementary Materials: The following supporting information can be downloaded at https: //www.mdpi.com/article/10.3390/metabo12020114/s1, Table S1: Quadratic model parameters including term estimates, standard error (Std.Error), $\mathrm{t}$ values and $p$-values. Term significance is provided in the column Signif, in a scale given at the footnote of the table. Extraction temperature (x1), conditioning time (x2), extraction time (x3), desorption time (x4) and desorption temperature (x5). Example: $x 1$ is a first order term, $x 1: x 2$ is a two-way interaction term and $x 1^{\wedge} 2$ is a quadratic term, Table S2: Quality characteristics of EVOO samples, Table S3: Complete Circumscribed Central Composite design indicating the run order along with the levels of each factor. Units for extraction temperature (Ex.temp) are ${ }^{\circ} \mathrm{C}$, conditioning time (Co.time) mins, extraction time (Ex.time) mins, desorption temperature (Des.temp) ${ }^{\circ} \mathrm{C}$ and desorption time (Des.time) mins, Figure S1: Contour plot for factors desorption temperature (Des.temp) and desorption time (Des.time). Green colour indicates lower response values and pink colour higher response values. Contours include corresponding response levels. The contour plot is a slice at the selected points for extraction temperature (Ex.temp) and extraction time (Ex.time), and the centre point for conditioning time (Co.time), Figure S2: PCA score plot showing the examined groups together with QC samples. QC samples are clustering together, showing system's stability, Figure S3: Geographical origin of Cretan EVOOs analysed in our study.

Author Contributions: A.L., I.S. and C.V., methodology, formal analysis, data curation, and writing-original draft preparation; V.T.P., A.S. and K.G.Z., Formal analysis, Writing—original draft; G.T., data curation, writing-original draft preparation, writing-review and editing, visualization, supervision and project administration. All authors have read and agreed to the published version of the manuscript.

Funding: This research was supported by the project FoodOmicsGR "Comprehensive Characterization of Foods" (MIS 5029057), which is implemented under the action "Reinforcement of the Research and Innovation Infrastructure", funded by the Operational Programme "Competitiveness, Entrepreneurship and Innovation" (NSRF 2014-2020) and co-financed by Greece and the European Union (European Regional Development Fund). All authors have read and agreed to the published version of the manuscript.

Institutional Review Board Statement: Not applicable.

Informed Consent Statement: Not applicable.

Data Availability Statement: Not applicable. 
Acknowledgments: We are grateful to the Sensory Evaluation Laboratory of Crete ACR in Rethymnon Crete, Greece for providing the organoleptic data of the VOO samples.

Conflicts of Interest: The authors declare no conflict of interest.

\section{References}

1. Arroyo-Manzanares, N.; Gabriel, F.; Carpio, A.; Arce, L. Use of Whole Electrophoretic Profile and Chemometric Tools for the Differentiation of Three Olive Oil Qualities. Talanta 2019, 197, 175-180. [CrossRef] [PubMed]

2. Conte, L.; Bendini, A.; Valli, E.; Lucci, P.; Moret, S.; Maquet, A.; Lacoste, F.; Brereton, P.; García-González, D.L.; Moreda, W.; et al. Olive Oil Quality and Authenticity: A Review of Current EU Legislation, Standards, Relevant Methods of Analyses, Their Drawbacks and Recommendations for the Future. Trends Food Sci. Technol. 2020, 105, 483-493. [CrossRef]

3. Da Silva, M.D.; Freitas, A.M.; Cabrita, M.J.; Garcia, R. Olive Oil Composition: Volatile Compounds; IntechOpen: London, UK, 2012; ISBN 978-953-307-921-9.

4. Commission Regulation (EEC) No 2568/91 of 11 July 1991 on the Characteristics of Olive Oil and Olive-Residue Oil and on the Relevant Methods of Analysis; European Union: Luxembourg, 1991; Volume 248.

5. Kalua, C.M.; Allen, M.S.; Bedgood, D.R.; Bishop, A.G.; Prenzler, P.D.; Robards, K. Olive Oil Volatile Compounds, Flavour Development and Quality: A Critical Review. Food Chem. 2007, 100, 273-286. [CrossRef]

6. Theodosi, S.; Kosma, I.S.; Badeka, A.V. Quality Characteristics of Koroneiki Olive Oil from Zakynthos Island (Greece) and Differentiation Depending on the Altitude Level. Eur. Food Res. Technol. 2021, 247, 1235-1248. [CrossRef]

7. Skiada, V.; Tsarouhas, P.; Varzakas, T. Comparison and Discrimination of Two Major Monocultivar Extra Virgin Olive Oils in the Southern Region of Peloponnese, According to Specific Compositional/Traceability Markers. Foods 2020, 9, 155. [CrossRef]

8. Revelou, P.-K.; Pappa, C.; Kakouri, E.; Kanakis, C.D.; Papadopoulos, G.K.; Pappas, C.S.; Tarantilis, P.A. Discrimination of Botanical Origin of Olive Oil from Selected Greek Cultivars by SPME-GC-MS and ATR-FTIR Spectroscopy Combined with Chemometrics. J. Sci. Food Agric. 2021, 101, 2994-3002. [CrossRef] [PubMed]

9. Pousinis, P.; Pechlivanis, A.; Lioupi, A.; Gika, H.; Virgiliou, C.; Kodra, D.; Marinaki, M.; Theodoridis, G. Innovation in Nutrition and Food Analysis: The FoodOmicsGR_Research Infrastructure. Column 2021, 17, 12-19.

10. Theodoridis, G.; Pechlivanis, A.; Thomaidis, N.S.; Spyros, A.; Georgiou, C.A.; Albanis, T.; Skoufos, I.; Kalogiannis, S.; Tsangaris, G.T.; Stasinakis, A.S.; et al. FoodOmicsGR_RI: A Consortium for Comprehensive Molecular Characterisation of Food Products. Metabolites 2021, 11, 74. [CrossRef]

11. Lioupi, A.; Nenadis, N.; Theodoridis, G. Virgin Olive Oil Metabolomics: A Review. J. Chromatogr. B 2020, 1150, 122161. [CrossRef]

12. Castro-Puyana, M.; Pérez-Míguez, R.; Montero, L.; Herrero, M. Reprint of: Application of Mass Spectrometry-Based Metabolomics Approaches for Food Safety, Quality and Traceability. TrAC Trends Anal. Chem. 2017, 96, 62-78. [CrossRef]

13. Reboredo-Rodríguez, P.; González-Barreiro, C.; Cancho-Grande, B.; Simal-Gándara, J. Dynamic Headspace/GC-MS to Control the Aroma Fingerprint of Extra-Virgin Olive Oil from the Same and Different Olive Varieties. Food Control. 2012, 25, 684-695. [CrossRef]

14. Aprea, E.; Gasperi, F.; Betta, E.; Sani, G.; Cantini, C. Variability in Volatile Compounds from Lipoxygenase Pathway in Extra Virgin Olive Oils from Tuscan Olive Germoplasm by Quantitative SPME/GC-MS. J. Mass Spectrom. 2018, 53, 824-832. [CrossRef]

15. Romero, I.; García-González, D.L.; Aparicio-Ruiz, R.; Morales, M.T. Validation of SPME-GCMS Method for the Analysis of Virgin Olive Oil Volatiles Responsible for Sensory Defects. Talanta 2015, 134, 394-401. [CrossRef]

16. Aprea, E.; Gika, H.; Carlin, S.; Theodoridis, G.; Vrhovsek, U.; Mattivi, F. Metabolite Profiling on Apple Volatile Content Based on Solid Phase Microextraction and Gas-Chromatography Time of Flight Mass Spectrometry. J. Chromatogr. A 2011, 1218, 4517-4524. [CrossRef]

17. Borges, T.H.; Ramalhosa, E.; Seiquer, I.; Pereira, J.A. Use of Response Surface Methodology (RSM) for the Identification of the Best Extraction Conditions for Headspace Solid-Phase Micro Extraction (HS-SPME) of the Volatile Profile of Cv. Arbequina Extra-Virgin Olive Oil. Eur. J. Lipid Sci. Technol. 2018, 120, 1700356. [CrossRef]

18. De los Angeles Fernandez, M.; Assof, M.; Jofre, V.; Silva, M.F. Volatile Profile Characterization of Extra Virgin Olive Oils from Argentina by HS-SPME/GC-MS and Multivariate Pattern Recognition Tools. Food Anal. Methods 2014, 7, 2122-2136. [CrossRef]

19. Wardencki, W.; Michulec, M.; Curyło, J. A Review of Theoretical and Practical Aspects of Solid-Phase Microextraction in Food Analysis. Int. J. Food Sci. Technol. 2004, 39, 703-717. [CrossRef]

20. Kiritsakis, A.K. Flavor Components of Olive Oil-A Review. J. Am. Oil Chem. Soc. 1998, 75, 673-681. [CrossRef]

21. Nunes, C.A.; Souza, V.R.; de Corrêa, S.C.; Silva, M.; de Cássia da Costa e Silva, M.; Bastos, S.C.; Pinheiro, A.C.M. Heating on the Volatile Composition and Sensory Aspects of Extra-Virgin Olive Oil. Ciênc. Agrotec. 2013, 37, 566-572. [CrossRef]

22. Morales, M.T.; Rios, J.J.; Aparicio, R. Changes in the Volatile Composition of Virgin Olive Oil during Oxidation: Flavors and Off-Flavors. J. Agric. Food Chem. 1997, 45, 2666-2673. [CrossRef]

23. Morales, M.T.; Przybylski, R. Olive Oil Oxidation. In Handbook of Olive Oil: Analysis and Properties; Aparicio, R., Harwood, J., Eds.; Springer US: Boston, MA, USA, 2013; pp. 479-522. ISBN 978-1-4614-7777-8.

24. Aparicio, R.; Morales, M.T. Characterization of Olive Ripeness by Green Aroma Compounds of Virgin Olive Oil. J. Agric. Food Chem. 1998, 46, 1116-1122. [CrossRef] 
25. Dhifi, W.; Angerosa, F.; Serraiocco, A.; Oumar, I.; Hamrouni, I.; Marzouk, B. Virgin Olive Oil Aroma: Characterization of Some Tunisian Cultivars. Food Chem. 2005, 93, 697-701. [CrossRef]

26. Angerosa, F. Influence of Volatile Compounds on Virgin Olive Oil Quality Evaluated by Analytical Approaches and Sensor Panels. Eur. J. Lipid Sci. Technol. 2002, 104, 639-660. [CrossRef]

27. Petronilho, S.; Lopez, R.; Ferreira, V.; Coimbra, M.A.; Rocha, S.M. Revealing the Usefulness of Aroma Networks to Explain Wine Aroma Properties: A Case Study of Portuguese Wines. Molecules 2020, 25, 272. [CrossRef] [PubMed]

28. Brkić Bubola, K.; Koprivnjak, O.; Sladonja, B.; Lukić, I. Volatile Compounds and Sensory Profiles of Monovarietal Virgin Olive Oils from Buža, Črna and Rosinjola Cultivars in Istria (Croatia). Food Technol. Biotechnol. 2012, 50, 192-198.

29. Cecchi, T.; Alfei, B. Volatile Profiles of Italian Monovarietal Extra Virgin Olive Oils via HS-SPME-GC-MS: Newly Identified Compounds, Flavors Molecular Markers, and Terpenic Profile. Food Chem. 2013, 141, 2025-2035. [CrossRef]

30. Mansouri, F.; Moumen, A.B.; Richard, G.; Fauconnier, M.-L.; Sindic, M.; Caid, H.S.; Elamrani, A. Flavor Profiles of Monovarietal Virgin Olive Oils Produced in the Oriental Region of Morocco. Oilseeds Fats Crops Lipids 2017, 24, A501. [CrossRef]

31. Kosma, I.S.; Kontominas, M.G.; Badeka, A.V. The Application of Chemometrics to Volatile Compound Analysis for the Recognition of Specific Markers for Cultivar Differentiation of Greek Virgin Olive Oil Samples. Foods 2020, 9, 1672. [CrossRef]

32. Angerosa, F.; Mostallino, R.; Basti, C.; Vito, R. Virgin Olive Oil Odour Notes: Their Relationships with Volatile Compounds from the Lipoxygenase Pathway and Secoiridoid Compounds. Food Chem. 2000, 68, 283-287. [CrossRef]

33. Reboredo-Rodríguez, P.; González-Barreiro, C.; Cancho-Grande, B.; Simal-Gándara, J. Improvements in the Malaxation Process to Enhance the Aroma Quality of Extra Virgin Olive Oils. Food Chem. 2014, 158, 534-545. [CrossRef]

34. Luna, G.; Morales, M.T.; Aparicio, R. Characterisation of 39 Varietal Virgin Olive Oils by Their Volatile Compositions. Food Chem. 2006, 98, 243-252. [CrossRef]

35. Pérez, A.G.; de la Rosa, R.; Pascual, M.; Sánchez-Ortiz, A.; Romero-Segura, C.; León, L.; Sanz, C. Assessment of Volatile Compound Profiles and the Deduced Sensory Significance of Virgin Olive Oils from the Progeny of Picual $\times$ Arbequina Cultivars J. Chromatogr. A 2016, 1428, 305-315. [CrossRef] [PubMed]

36. Stilo, F.; Cordero, C.; Sgorbini, B.; Bicchi, C.; Liberto, E. Highly Informative Fingerprinting of Extra-Virgin Olive Oil Volatiles: The Role of High Concentration-Capacity Sampling in Combination with Comprehensive Two-Dimensional Gas Chromatography. Separations 2019, 6, 34. [CrossRef]

37. Youssef, O.; Guido, F.; Manel, I.; Youssef, N.B.; Luigi, C.P.; Mohamed, H.; Daoud, D.; Mokhtar, Z. Volatile Compounds and Compositional Quality of Virgin Olive Oil from Oueslati Variety: Influence of Geographical Origin. Food Chem. 2011, 124, 1770-1776. [CrossRef]

38. Cerretani, L.; Salvador, M.D.; Bendini, A.; Fregapane, G. Relationship Between Sensory Evaluation Performed by Italian and Spanish Official Panels and Volatile and Phenolic Profiles of Virgin Olive Oils. Chem. Percept. 2008, 1, 258. [CrossRef]

39. Gilbert-López, B.; Robles-Molina, J.; García-Reyes, J.F.; Molina-Díaz, A. Rapid Determination of BTEXS in Olives and Olive Oil by Headspace-Gas Chromatography/Mass Spectrometry (HS-GC-MS). Talanta 2010, 83, 391-399. [CrossRef]

40. Bempelou, E.; Anagnostopoulos, C.; Liapis, K. Investigation of Naphthalene Contamination in Olive Oil from Greece. Toxicol. Environ. Chem. 2019, 101, 45-58. [CrossRef]

41. Nenadis, N.; Mastralexi, A.; Tsimidou, M.Z. Physicochemical Characteristics and Antioxidant Potential of the Greek PDO and PGI Virgin Olive Oils (VOOs). Eur. J. Lipid Sci. Technol. 2019, 121, 1800172. [CrossRef]

42. Kosma, I.; Vatavali, K.; Kontakos, S.; Kontominas, M.; Kiritsakis, A.; Badeka, A. Geographical Differentiation of Greek Extra Virgin Olive Oil from Late-Harvested Koroneiki Cultivar Fruits. J. Am. Oil Chem Soc. 2017, 94, 1373-1384. [CrossRef]

43. Pouliarekou, E.; Badeka, A.; Tasioula-Margari, M.; Kontakos, S.; Longobardi, F.; Kontominas, M.G. Characterization and Classification of Western Greek Olive Oils According to Cultivar and Geographical Origin Based on Volatile Compounds. J. Chromatogr. A 2011, 1218, 7534-7542. [CrossRef]

44. Lukić, I.; Lukić, M.; Žanetić, M.; Krapac, M.; Godena, S.; Brkić Bubola, K. Inter-Varietal Diversity of Typical Volatile and Phenolic Profiles of Croatian Extra Virgin Olive Oils as Revealed by GC-IT-MS and UPLC-DAD Analysis. Foods 2019, 8, 565. [CrossRef] [PubMed]

45. Bajoub, A.; Sánchez-Ortiz, A.; Ajal, E.A.; Ouazzani, N.; Fernández-Gutiérrez, A.; Beltrán, G.; Carrasco-Pancorbo, A. First Comprehensive Characterization of Volatile Profile of North Moroccan Olive Oils: A Geographic Discriminant Approach. Food Res. Int. 2015, 76, 410-417. [CrossRef] [PubMed]

46. Şişik Oğraş, Ş.; Kaban, G.; Kaya, M. Volatile Compounds of Olive Oils from Different Geographic Regions in Turkey. Int. J. Food Prop. 2018, 21, 1833-1843. [CrossRef]

47. Vichi, S.; Guadayol, J.M.; Caixach, J.; López-Tamames, E.; Buxaderas, S. Monoterpene and Sesquiterpene Hydrocarbons of Virgin Olive Oil by Headspace Solid-Phase Microextraction Coupled to Gas Chromatography/Mass Spectrometry. J. Chromatogr. A 2006, 1125, 117-123. [CrossRef] [PubMed]

48. Zunin, P.; Boggia, R.; Salvadeo, P.; Evangelisti, F. Geographical Traceability of West Liguria Extravirgin Olive Oils by the Analysis of Volatile Terpenoid Hydrocarbons. J. Chromatogr. A 2005, 1089, 243-249. [CrossRef]

49. Mansour, A.B.; Gargouri, B.; Flamini, G.; Bouaziz, M. Effect of Agricultural Sites on Differentiation between Chemlali and Neb Jmel Olive Oils. J. Oleo. Sci. 2015, 64, 381-392. [CrossRef] 
50. Kandylis, P.; Vekiari, A.S.; Kanellaki, M.; Grati Kamoun, N.; Msallem, M.; Kourkoutas, Y. Comparative Study of Extra Virgin Olive Oil Flavor Profile of Koroneiki Variety (Olea Europaea Var. Microcarpa Alba) Cultivated in Greece and Tunisia during One Period of Harvesting. LWT Food Sci. Technol. 2011, 44, 1333-1341. [CrossRef]

51. Angerosa, F.; Servili, M.; Selvaggini, R.; Taticchi, A.; Esposto, S.; Montedoro, G. Volatile Compounds in Virgin Olive Oil: Occurrence and Their Relationship with the Quality. J. Chromatogr. A 2004, 1054, 17-31. [CrossRef]

52. Ribeiro, L.H.; Costa Freitas, A.M.; Gomes da Silva, M.D.R. The Use of Headspace Solid Phase Microextraction for the Characterization of Volatile Compounds in Olive Oil Matrices. Talanta 2008, 77, 110-117. [CrossRef]

53. Wenig, P.; Odermatt, J. OpenChrom: A Cross-Platform Open Source Software for the Mass Spectrometric Analysis of Chromatographic Data. BMC Bioinform. 2010, 11, 405. [CrossRef]

54. Brocks, J.J.; Hope, J.M. Tailing of Chromatographic Peaks in GC-MS Caused by Interaction of Halogenated Solvents with the Ion Source. J. Chromatogr. Sci. 2014, 52, 471-475. [CrossRef] [PubMed]

55. Ihaka, R.; Gentleman, R. R: A Language for Data Analysis and Graphics. J. Comput. Graph. Stat. 1996, 5, 299-314. [CrossRef]

56. RStudio I Open Source \& Professional Software for Data Science Teams. Available online: https://rstudio.com/ (accessed on 17 August 2021).

57. Grömping, U. R Package FrF2 for Creating and Analyzing Fractional Factorial 2-Level Designs. J. Stat. Softw. 2014, 56, 1-56. [CrossRef]

58. Grömping, U. R Package DoE.Base for Factorial Experiments. J. Stat. Softw. 2018, 85, 1-41. [CrossRef]

59. Lenth, R.V. Response-Surface Methods in R., Using Rsm. J. Stat. Softw. 2009, 32, 1-17. [CrossRef] 